\title{
Using experimental evolution to understand the persistence of bet-hedging traits in Saccharomyces cerevisiae
}

by

Shravan Raghu

A thesis submitted to the Faculty of Graduate and Postdoctoral Affairs in partial fulfillment of the requirements for the degree of

Master of Science

in

Biology

Carleton University

Ottawa, Ontario

C2019, Shravan Raghu 


\section{Abstract}

Bet hedging, as a mode of evolutionary response, maximizes long-term fitness in unpredictably fluctuating environments by sacrificing fitness in average environments in exchange for increased fitness in rare but calamitous environments. A bet-hedging strategy is suboptimal over short timescales, but optimal over long timescales because it buffers temporal variance in fitness. It is unclear how such strategies can persist when selection is expected to improve suboptimal traits in the short-term. It has been proposed that the maintenance of bet hedging is possible if the ability to adaptively track environments is constrained in the short-term. To test this hypothesis, we first develop an experimental system using Saccharomyces cerevisiae that is suitable for the evolution of bet hedging under fluctuating selection. In this system, fluctuating selection is imposed through repeated heat shocks separated by intervening benign environments. Using this system, we evolve bet hedging under fluctuating selection and ask whether it has evolved as a constraint on adaptive tracking. We do this by selecting for the retention of the bet-hedging trait through continual reduction in the frequency of extreme environments across two sequential evolution regimes, viz. Regime-A (high frequency of heat shocks) and Regime-B (low frequency of heat shocks). To measure evolved constraints, we further evolve lines from both regimes under extended benign conditions and assay for the loss of heat shock tolerance. We observe the retention of heat shock tolerance despite selection under extended benign conditions in lines from Regime-B but not in lines from Regime-A. Moreover, we find that evolution under 
extended benign conditions results in increased fitness under benign conditions in lines from Regime-A but not in lines from Regime-B. Taken together, these results suggest that a constraint on adaptive tracking evolves in lines from Regime-B relative to lines from Regime-A. While constraints have traditionally been perceived to be maladaptive, we show that they may be adaptive and could potentially be selected for in the long term. 


\section{Acknowledgements}

While this thesis may appear to be the effort of one person, it is anything but. I would first like to thank my supervisors Dr. Andrew Simons and Dr. Myron Smith for giving me the opportunity to work with them. Andrew has been a wonderful mentor and guide through my masters. Meetings with Andrew, from short updates to long gruelling brain storming sessions, always left me reinvigorated and with a sense of purpose. Myron has been an inspiring and patient guide throughout my masters. His support in the lab is only rivalled by his support outside it. Thank you for the endless supply of hot peppers, hot sauces and honey. The Department of Biology at Carleton University, especially the administrative staff, has my gratitude for all their help.

I would like to thank my committee members Dr. Alex Wong and Dr. Jessica Forrest for their immensely helpful comments and insights. I would also like to thank Maryam Hajikarimlou for her help and for providing yeast strains. I would like to thank members of the Smith lab-Tom Witte, Emma Micalizzi, Ghazaleh Nourparvar, Bodunde Oyetoran, Fatima Haider and Chanchal Yadav for their support and help around the lab. It has truly been a privilege to work alongside the amazing members of the Simons labHarry Hitsman, Winston Campeau, Lina Wen and Riley Morris.

I would like to thank my parents, Raghu and Hema, and my sister, Shruthi, for their unwavering support. I would also like to thank my friends Sheryl, Lakshmi, Adi, Karan, Prasanth, Vinod, Hemant, Nidhin and Ananya for their encouragement, advice 
and support. Finally, I would like to thank S. N. Goenka and the Vipassana foundation (especially Dhamma Suttama) for everything that they have done for me. 


\section{Table of contents}

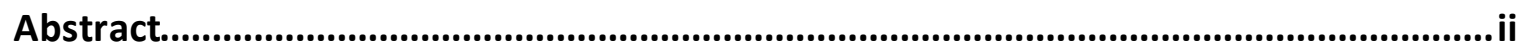

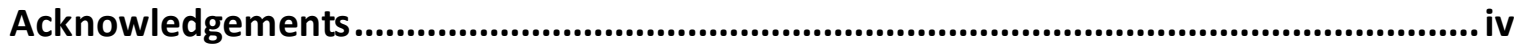

Table of contents ................................................................................................................... vi

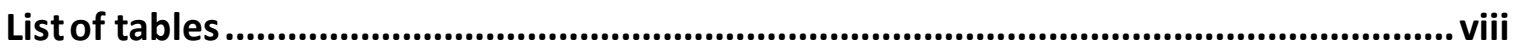

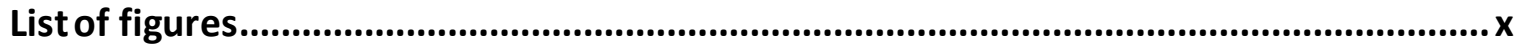

List of Appendices....................................................................................................................

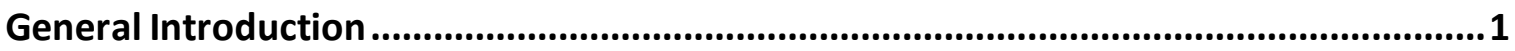

Chapter 1: Developing an experimental system suitable for the evolution of bet

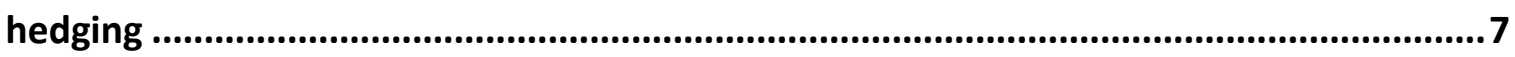

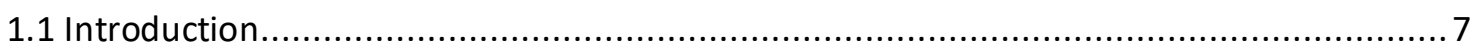

1.1.1 Antifungal resistance - Salt tolerance trade-off................................................ 11

1.1.2 Heat Shock Tolerance-Growth trade-off ..................................................... 14

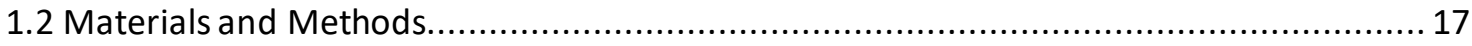

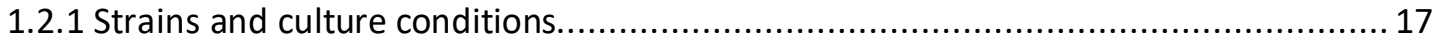

1.2.2 Preparation of microtitration plates of fluconazole and $\mathrm{NaCl}: \ldots \ldots \ldots \ldots \ldots \ldots \ldots \ldots \ldots . . . \ldots 18$

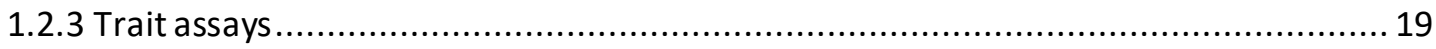

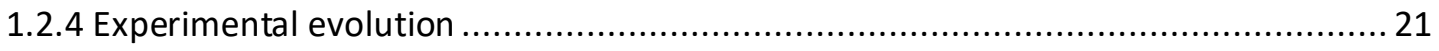

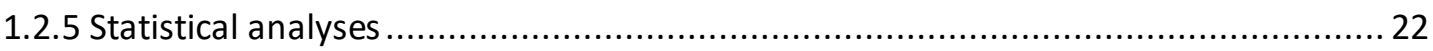

1.3 Results 23

1.3.1 Fluconazole resistance $-\mathrm{NaCl}$ tolerance trade-off 23

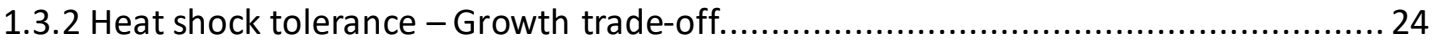

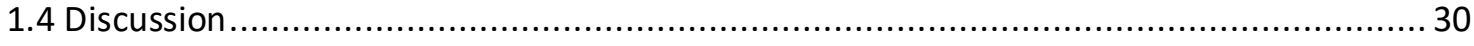

Chapter 2: Bet hedging evolves as a constrainton adaptation.......................................35

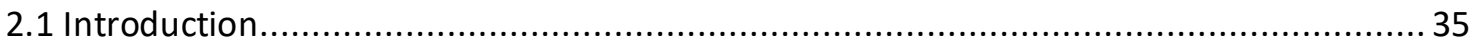

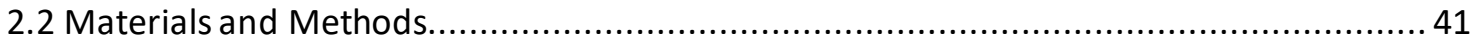

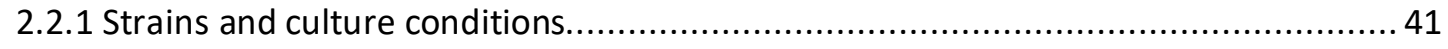

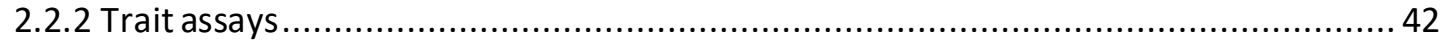

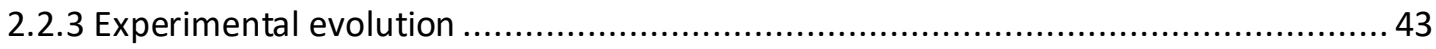

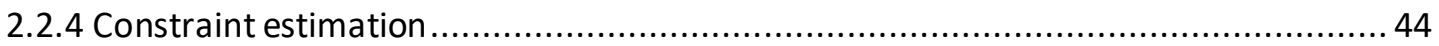

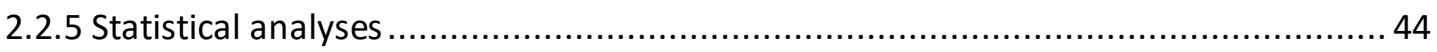


2.3.1 Heat shock tolerance evolves at the cost of fitness at $30{ }^{\circ} \mathrm{C}$ in both regimes. 45

2.3.2 Analysis of the evolutionary constraint assay .46

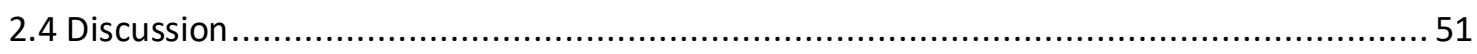

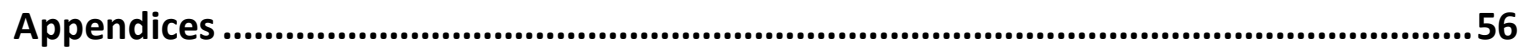

Appendix A: Heat shock tolerance across phases of yeast growth................................. 56

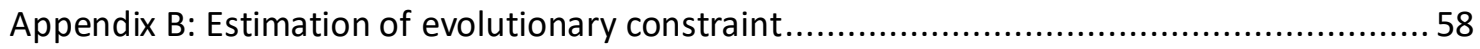

Appendix C: Estimation of doubling time and number of generationsper transfer ...............60 60

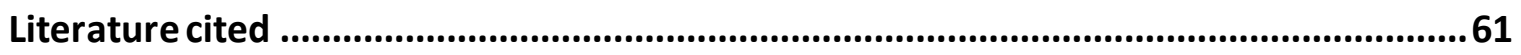




\section{List of tables}

Table 1.1: Results of a One-Way ANOVA for differences in fluconazole MICs across strains. The strains compared were S288C, ERG3 $\Delta$, Ev1 and Ev2

Table 1.2: Results of a One-Way ANOVA for differences in $\mathrm{NaCl}$ MICs across strains. The strains compared were S288C, ERG3 $\Delta$, Ev1and Ev2

Table 1.3: Post-hoc Tukey test results for differences in fluconazole MICs across strains. Common letters indicate homogenous subsets. Letters that are not shared indicate significantly different mean values

Table 1.4: Post-hoc Tukey test results for differences in $\mathrm{NaCl}$ MICs across strains. Common letters indicate homogenous subsets. Letters that are not shared indicate significantly different mean values

Table 1.5: Results of a One-Way ANOVA for differences in heat shock tolerance across strains. The strains compared were S288C, T1 and Iso-1.

Table 1.6: Post-hoc Tukey test results for differences in heat shock tolerance across strains. Common letters indicate homogenous subsets. Letters that are not shared indicate significantly different mean values

Table 1.7: Results of a One-Way ANOVA for differences competitive fitness across strains. The strains compared were S288C, T1 and Iso-1.

Table 1.8: Post-hoc Tukey test results for differences in competitive fitness across strains. Common letters indicate homogenous subsets. Letters that are not shared indicate significantly different mean values

Table 2. 1: Results of a one-way ANOVA for differences in heat shock tolerance across evolved lines from EoR-A \& EoR-B and theT1 ancestor 
Table 2. 2: Post-hoc Tukey test results for differences in heat shock tolerance across evolved lines and the T1 ancestor. Common letters indicate homogenous subsets. Letters that are not shared indicate significantly differentmean values

Table 2. 3: Results of a one-way ANOVA for differences in relative fitness under benign conditions across evolved lines from EoR-A \& EoR-B and the T1 ancestor

Table 2. 4: Post-hoc Tukey test results for differences in relative fitness under benign conditions across evolved lines and the T1 ancestor. Common letters indicate homogenous subsets. Letters that are not shared indicate significantly different mean values

Table 2. 5: Results of a two-factor ANOVA for differences in heat shock tolerance across evolution regimes and time points. Lines from EoR-A and EoR-B and time points at generation 0,100 and 150 of the evolutionary constraint assaywere compared

Table 2. 6: Results of a post-hoc Tukey test for differences in heat shock tolerance at different timepoints for lines from EoR-A and EoR-B. Common letters indicate homogenous subsets. Letters that are not shared indicate significantly different mean values.....

Table 2. 7: Results of a two-factor ANOVA for differences in fitness at $30^{\circ} \mathrm{C}$ across evolution regimes and time points. Lines from EoR-A and EoR-B and time points at generation 0 and 150 of the evolutionary constraint assaywere compared

Table 2. 8: Results of a post-hoc Tukey test for differences in fitness at $30^{\circ} \mathrm{C}$ at different timepoints for lines from EoR-A and EoR-B. Common letters indicate homogenous subsets. Letters that are not shared indicate significantly differentmean values 


\section{List of figures}

Figure 1.1: Relative yield of S288C, ERG3 $\triangle$ strain, Ev1 and Ev2 across (A) fluconazole and (B) $\mathrm{NaCl}$ gradients. Red boxes denote MIC values for each strain (placed above highest concentration tested if growth inhibition does not occur across entire range). Yield values across concentration gradient are relative to agent-free controls (bottomcells).

Figure 1.2: (A) Heat shock tolerance (measured as \% Survival) and (B) competitive fitness of S288C, T1 and Iso-1. Errorbars represent SD

Figure 2.1: Four possible scenarios representing the outcome of assays of evolved constraints in lines from Regime-B (blue; low-frequency extreme events) relative to those from Regime-A (red; high-frequency extreme events). Scenarios A and Brepresent the null prediction where a constraint does not evolve in Regime-B relative to Regime-A, i.e., heat shock tolerance is lost at similar rates. Scenarios $C$ and $D$ represent the evolution of constraint in lines from Regime-B relative to lines from Regime-A. This is characterized by the lower evolutionary rate of loss of heat shock tolerance in Regime-B lines relative to Regime-A lines

Figure 2.2: Trait measurements after evolution in Regime-A and Regime-B relative to the $\mathrm{T} 1$ ancestor. (A) Heat shock tolerance (measured as \% Survival) across lines. (B) Competitive fitness across lines. Errorbarsrepresent SD.

Figure 2.3: Evolved constraints measured as (A) loss of heat shock tolerance (measured as $\%$ Survival) and (B) gain in competitive fitness at $30^{\circ} \mathrm{C}$ after selection in the evolutionary constraint assay under benign conditions for 150 generations in lines from Regime-A (red) and Regime-B (blue). Errorbarsrepresent SD 


\section{List of Appendices}

Appendix A: Heat shock tolerance across phases ofyeast growth ..............................54

Appendix B: Estimation of evolutionary constraint...............................................56

Appendix C: Estimation of doubling time and number of generations pertransfer .........58 


\section{General Introduction}

Bet hedging, as a mode of evolutionary response, is a risk-aversion strategy that maximizes long-term fitness in unpredictably fluctuating environments (Lewontin \& Cohen, 1969; Philippi \& Seger, 1989). A bet-hedging strategy results in increased fitness in detrimental/extreme environments at the cost of fitness in average or expected environments. Because extreme events tend to be rare, there is strong selection to improve fitness of suboptimal phenotypes over the short-term in average environments. The central focus of this thesis is to address how bet hedging evolves when it entails selection for apparent suboptimality over shorter timescales.

In addition to bet hedging, adaptive tracking and phenotypic plasticity may also be selected for in fluctuating environments (Simons, 2011). The reliability of environmental cues and the timescale of environmental variation determine these modes of evolutionary response (Botero, Weissing, Wright, \& Rubenstein, 2015). Environmental cues that signal impending changes often precede environmental conditions. The reliability of these cues indicate how well correlated these cues are with changes in the environment (Reed, Robin, Schindler, Hard, \& Kinnison, 2010; Schmitt, McCormac, \& Smith, 1995; Stabell, Ogbebo, \& Primicerio, 2003). An example of a reliable cue is change in photoperiod which serves as an indicator of seasonally varying temperature. The timescale of environmental variation is determined by the number of generations between environmental events. Bet hedging is expected to evolve when environmental fluctuations are frequent and when environmental cues are unreliable. 
Evolutionary response through adaptive tracking requires genetic variation for fitness, and occurs if environmental fluctuations are relatively infrequent regardless of the predictability of environmental cues. Because it is limited by heritability, adaptation always lags behind a change in the environment, and frequent environmental fluctuations constrain the effectiveness of adaptive tracking (Simons, 2011). In rapidly fluctuating environments, if reliable cues exist, phenotypic plasticity is expected to evolve (Botero, Weissing, Wright, \& Rubenstein, 2015).

To understand how bet hedging serves as a risk spreading strategy, it is necessary to scrutinize how fitness is interpreted through time. The simplest way to condense fitness values for an individual or genotype over a period of time is by representing it as the arithmetic mean fitness (AMF) of all fitness values over time. However, utilizing the AMF to infer evolutionary success can be problematic when variance in fitness of a genotype exists through time. Fitness through time can also be represented by the geometric mean fitness (GMF) which is the $\mathrm{n}^{\text {th }}$ root of the product of $\mathrm{n}$ fitness values. The GMF is a more appropriate measure of fitness over time than the AMF and this can be understood from three perspectives. Firstly, since reproduction and changes in gene frequency in a population are both multiplicative processes, it makes more sense intuitively to use the GMF. Secondly, it may be demonstrated in simple mathematical terms that the arithmetic mean fitness (AMF) as a measure does not represent success over generations under temporal fluctuations in fitness. It has been shown that long-term evolutionary success is a function of both the mean and variance in fitness (Dempster, 1955). This long-term evolutionary success is better explained bya 
high GMF as opposed to a high AMF because small fitness values do not greatly affect (and are, thus, not adequately represented in) the AMF as much as they do the GMF (Gillespie, 1977; Orr, 2009). Finally, because the GMF is equivalent to the AMF when there is no variance in fitness, the AMF may be considered a special case of the GMF. Therefore, a genotype/allele that predominates over time has maximized the GMF. Traits that maximize the GMF, therefore, are associated with low fitness variance (Lee \& Doughty, 2003; Yoshimura, Tanaka, Togashi, Iwata, \& Tainaka, 2009). A bet-hedging strategy does exactly that - it minimizes the risk of low fitness in an environment by reducing the fitness variance across all environments. This may be visualized by contrasting a specialist strategy against a bet-hedging strategy in fluctuating benign and detrimental environments. The specialist strategy does better in benign environments but, consequently, much worse in detrimental environments compared to the bethedging strategy. The low fitness values in detrimental environments severely depresses the overall GMF. In contrast, the fitness values of the bet-hedger in both environments do not differ drastically. As a result, low fitness values are avoided in detrimental environments at the expense of high fitness values in benign environments (Simons, 2011). Fluctuations in fitness exist when adaptation to all environments has not occurred. This may be due to across-environment trade-offs in resource allocation, antagonistic pleiotropy, etc. Under these conditions, adaptation to one environment comes at the cost of fitness in another environment. If these trade-offs do not exist, fitness values will not variably fluctuate across environments and maximizing the GMF 
may occur through adaptive tracking (i.e. would be equivalent to AMF), and not depend on the evolution of bet hedging.

Risk avoidance (or conservative bet hedging) and risk spreading (or diversification bet hedging) represent the two broad classes of bet-hedging strategies. The bet-hedging strategy described in the scenario above operates through risk avoidance, a type of conservative bet hedging that results in 'middle-of-the-road' traits characterized by suboptimal but safe trait values. However, risk minimization can also be achieved through risk spreading. Diversification bet hedging operates by spreading risk among individuals of a given genotype (Simons, 2009); thus, non-zero survival is ensured across environments (Childs, Metcalf, \& Rees, 2010).

Both conservative and diversification bet hedging maximize long-term fitness by reducing the fitness variance across environments resulting in a suboptimal phenotype in average or expected environments. Because detrimental events tend to be rare, strong selective pressures exist in the short-term to improve fitness for suboptimal phenotypes in average environments. Research has focussed on whether or not bet hedging evolves under given conditions of fluctuating selection. However, surprisingly little attention has been focussed on how bet hedging can evolve when it entails, by definition, selection for apparent suboptimality over shorter time scales. It has been proposed in a conceptual model that bet hedging evolves as a constraint on adaptive tracking. An evolutionary constraint is a limitation on the course of adaptation and has traditionally been viewed as a hinderance to natural selection. A bet-hedging trait that is suboptimal in average environments can be maintained despite strong selection to 
improve this trait if bet hedging evolves as a constraint on adaptive tracking. When adaptation to average environments is constrained, fitness in rare but detrimental environments is not compromised resulting in increased long-term fitness. In theory, this constraint would be continually reinforced by selection during the rare but severe events. In this thesis, we take an experimental evolution approach using the yeast Saccharomyces cerevisiae to test the hypothesis that bet hedging evolves as a constraint on adaptive tracking. Our test involved two steps:

1) Developing an experimental system using Saccharomyces cerevisiae that would allow for the evolution of bet hedging under fluctuating selection.

2) Utilizing this system to empirically test the hypothesis that bet hedging evolves as a constraint on adaptive tracking.

The objective of Chapter 1 is to identify and develop the experimental system and assess fitness trade-offs between environments. Fitness trade-offs are central to the evolution of bet hedging as they generate variance in fitness across environments. Recall that without trade-offs, maximizing long-term fitness will not depend on bet hedging but rather on the independent optimization of all traits under selection. In this first chapter, two separate trade-off systems are assessed. In the first system, we assess trade-offs between antifungal resistance to the azole antifungal fluconazole, and salt tolerance using sodium chloride. In the second system, we assess trade-offs between heat shock tolerance and growth under benign conditions. 
The objective of Chapter 2 is to empirically test the hypothesis that bet hedging evolves as a constraint on adaptive tracking using the heat shock tolerance system developed in Chapter 1 . We do this by selecting for the retention of the bet-hedging trait through continual reduction in the frequency of extreme environments across two sequential evolution regimes. As the frequency of extreme environments decrease, selective pressure to enhance fitness under benign conditions increases. However, due to fitness trade-offs, any increase in fitness under benign conditions comes at the cost of survival under extreme environments. Therefore, if there is nonzero genetic variation for constraint, this will be recurrently selected for during the extreme environments, and we expect the evolution of a constraint on adaptive tracking under benign conditions; in other words, evolution of a phenotype with the ability to retain a sufficient level of survival under extreme environments despite increased selective pressure to improve fitness under benign conditions. Due to Chapter 2 being prepared for publication, some redundancy occurs among introductory sections.

Bet hedging studies are diverse both in terms of the types of studies (computational, experimental, field studies) and experimental systems used. Despite this, bet-hedging theory lacks a mechanistic explanation. Although proximate mechanisms for bet hedging have been proposed, they pertain to the specific organism and the evolution of the trait in question rather than to the evolution of bet hedging.

The goal of this study is to bridge this important gap in knowledge by testing the idea that bet-hedging traits evolve as a constraint on adaptive tracking. 


\section{Chapter 1: Developing an experimental system suitable for the evolution of bet hedging}

\subsection{Introduction}

A surge of interest in recent years has led to growing empirical evidence for bet hedging (Frankenhuis, Panchanathan, \& Belsky, 2016; Furness, Lee, \& Reznick, 2015; García-Roger, Serra, \& Carmona, 2014; Graham, Smith \& Simons, 2014; Gremer, Kimball, \& Venable, 2016; Lycus et al., 2018; Tarazona, García-Roger \& Carmona 2017). However, tests of bet hedging are difficult, and variation in the strength of evidence exists. A system to categorize tests of candidate bet-hedging traits proposed by Simons (2011) posits that strong evidence for bet hedging is conditional on demonstrating that bet hedging maximizes the geometric-mean fitness (GMF) over fluctuating selection. Bet hedging is adaptive over the long-term but, it can appear maladaptive in the short term (Seger, 1987; Simons, 2011). Because of this, specialist strategies that are only beneficial in the short-term can be misconstrued to result in long-term evolutionary success. Therefore, long-term studies are required to test if a candidate bet-hedging trait is indeed adaptive, which at least partially explains the lack of strong quantitative evidence for bet hedging.

Experimental evolution, as a research tool, is ideal for the study of bet hedging as it allows for long-term environmental manipulations that may capture evolutionary effects of naturally fluctuating environments. Furthermore, since this approach allows 
for the study of underlying evolutionary processes in real time and in replicate 
populations, diverse questions can be addressed including the presence of evolutionary costs or trade-offs between traits; constraints on evolution, and changes in population genetic parameters (Kawecki et al., 2012). For these reasons, experimental evolution is being widely adopted to address questions pertaining to bet hedging using a variety of study systems from microbes to mammals (Beaumont, Gallie, Kost, Ferguson, \& Rainey, 2009; Bradford \& Roff, 1993; Graham, Smith, \& Simons, 2014; Rainey et al., 2011; Rajon, Desouhant, Chevalier, Débias, \& Menu, 2014; Tarazona, García-Roger, \& Carmona, 2017; Zammuto \& Millar, 1985).

When asking general questions about evolutionary processes that apply broadly, it is largely a matter of convenience as to which experimental organism is best suited for a particular evolutionary problem. Microbial model systems are widely used for experimental evolution due to their short generation times and large population sizes, allowing for faster adaptation (Elena \& Lenski, 2003; Kassen, 2009). The focus of this thesis is to address how bet hedging is evolves when it entails selection for apparent suboptimality in the short-term. We hypothesize that this is possible if bet hedging evolves as a constraint on adaptation, and test this using the Saccharomyces cerevisiae model system. Recent large-scale phenotype screens and phenome analyses (Engel et al., 2009; Ferea, Botstein, Brown, \& Rosenzweig, 1999; Fernandez-Ricaud et al., 2007; Suzuki et al., 2018; Warringer \& Blomberg, 2003; Yoshikawa et al., 2011) coupled with a wide range of genetic and genomic tools (Dunham et al., 2002; Leu \& Murray, 2006; Ratcliff, Denison, Borrello, \& Travisano, 2012; Segrè, Murray, \& Leu, 2006) have made the budding yeast an ideal choice for experimental evolution. The strain of 
Saccharomyces cerevisiae used in this study is the S288C laboratory strain designed by Mortimer and Johnston in 1986. This strain is a haploid, has only a few nutritional requirements and can be maintained to reproduce asexually as it is unable to switch its mating type (Louis, 2016; Mortimer \& Johnston, 1986).

To test the hypothesis that bet hedging evolves as a constraint on adaptive tracking, it is important to develop a system that would allow for the evolution of a bethedging trait. Bet hedging is expected to evolve under unpredictably fluctuating environments. In its simplest form, this would entail an evolution regime comprising two alternating environmental states- stressful and benign (Cohen, 1966). In previous studies, unpredictability has been incorporated into microbial evolution regimes by randomizing the order of benign and stressful environments (Graham, Smith \& Simons, 2014; Karve, Bhave, \& Dey, 2018). In contrast to randomization, we believe that alternating between these environmental states is appropriate for the present circumstances. In our system, transfers between environmental states occurs at the saturation phase of growth, i.e., there are $\sim 8$ generations between environmental states. This results in an almost complete population turnover and individuals in a given environment are largely underrepresented in following environments. Therefore, an overwhelming number of individuals in any environment have no direct experience of past environments, making future environments unpredictable. In further support for this argument, the previously discussed study by Karve, Bhave \& Dey (2018) found no difference in the extent of adaptation when environmental states in evolution regimes were either alternated or randomized. 
The evolution of bet hedging also requires fitness trade-offs between environments. Evolutionary trade-offs are thought to be instrumental to the maintenance of biological diversity both within and among species (Clark et al., 2007). Based on the number of traits involved, trade-offs can be categorized into two types. A one-trait trade-off exists when the direction of selection differs for a single trait due to differences in environments or other selective agents. An example of this trade-off is the case of stabilizing selection acting on gall size produced by gall fly larvae in goldenrod. While larger galls prevent ovipositors of parasitoid wasps from reaching flies, they also are more visible and, therefore, susceptible to attack by birds (Weis \& Gorman, 1990). A multi-trait trade-off arises when selection to improve two or more traits is constrained due to a common limiting resource. It is important to note that when a common resource is abundant, selection can act to simultaneously improve all traits in question. A multi-trait trade-offis only manifested when a common resource becomes limiting. An example of a multi-trait trade-off is one between offspring size and offspring number (Agrawal, Conner, \& Rasmann, 2010; McGinley \& Charnov, 1988). Trade-offs exist when adaptation to two environments cannot proceed simultaneously, such that one trait is improved at the cost of another or due to competing demands on a single trait. Hence, trait specialization in a given environmental state can result in low fitness values when environmental states change. Under such environmental fluctuations, bet hedging is a potentially beneficial strategy in the long-term. This is because bet hedging maximizes GMF by avoiding low fitness. In contrast, if trade-offs do not exist, the strategy that 
would maximize the GMF would simply be the improvement of all traits under selection (Stearns, 1989).

Therefore, to empirically test our hypothesis, both components, viz., unpredictable environmental fluctuation and trade-offs, need to be incorporated in the selection regime. The following sections detail two systems that were assessed for fitness trade-offs. The first system investigates the trade-off between antifungal resistance and salt tolerance and the second system investigates the trade-off between heat shock tolerance and growth.

\subsubsection{Antifungal Resistance - Salt Tolerance Trade-off}

To combat the growing threat of pathogenic fungal infections, a variety of antifungal drugs have been developed. These vary in their mode of action ranging from fungistatic drugs (that slow growth enough for immune functions to overcome infection) to fungicidal drugs (that directly kill the fungal source of infection). However, only a few different classes of antifungals are routinely used to control pathogenic fungi in animal systems and most of these either directly target ergosterol or the ergosterol biosynthetic pathway (Anderson et al., 2003). Ergosterol is an important and abundant component of the fungal cell membrane whose functions include the maintenance of membrane integrity and fluidity and ensuring the correct functioning of membranebound enzymes (Cowen, Sanglard, Howard, Rogers, \& Perlin, 2014; Lupetti, Danesi, 
Campa, Tacca, \& Kelly, 2002). Moreover, ergosterol is not found in animal cells, making it an ideal antifungal target. The drug used in this study, fluconazole, belongs to the azole class of antifungals that targets the ergosterol biosynthetic pathway.

In the last decade, the widespread use of antifungal drugs has directly contributed to the increased incidence of drug-resistant pathogenic fungi (Perlin, Rautemaa-Richardson, \& Alastruey-Izquierdo, 2017; Rogers, 2002). These drug-resistant pathogenic fungi have also been observed in environmental reservoirs where medically relevant antifungals have been used in agricultural practices (Verweij, Snelders, Kema, Mellado, \& Melchers, 2009). However, evolved antifungal drug resistance is generally assumed to come at a physiological cost resulting in decreased fitness in the absence of the drug (Ben-Ami et al., 2011; Hill, O’Meara, \& Cowen, 2015; Huang \& Kao, 2012; Vincent, Lancaster, Scherz-Shouval, Whitesell, \& Lindquist, 2013). This has also been observed in the case of evolved fluconazole resistance in yeasts (Anderson et al., 2003; Sasse et al., 2012).

Resistance to fluconazole can evolve in multiple ways including: (i) upregulation of efflux pumps (usually through mutations in PDR1, PDR3, PDR5 and MDR1) to reduce intracellular concentrations of the drug, (ii) upregulation of the azole drug target, primarily through the increased transcription of ERG11, (iii) decreased binding affinity of the drug to the target via mutations in ERG11 that encodes for a cytochrome P450 used in the ergosterol biosynthetic pathway, and (iv) altered sterol composition through the inactivation of $E R G 3$ which is responsible for the formation of the toxic product $14 \alpha$ - 
methyl-3,6-diol from $14 \alpha$-methylfecosterol in the ergosterol biosynthetic pathway. Inactivation of ERG3 results in formation of a nontoxic precursor of ergosterol and a suboptimal but functional cell membrane (Kanafani \& Perfect, 2008; Lupetti et al., 2002). These differing paths that all lead to fluconazole resistance can each potentially come with different costs.

We used two approaches to study fitness trade-offs associated with fluconazole resistance. In our first approach we used an artificially constructed yeast strain that had the $E R G 3$ gene deleted (ERG3 $)$ to assess trade-offs associated with fluconazole resistance. This deletion essentially represents a loss-of-function mutation in ERG3 which confers fluconazole resistance. To assay fluconazole resistance, we performed Minimum Inhibitory Concentration (MIC) tests using S288C and the ERG3A strain to compare relative growth across a fluconazole gradient.

The yeast cell membrane is important for the maintenance of the internal cellular environment and, hence, plays a vital role in the cellular stress response. Since the $E R G 3 \Delta$ strain has an altered sterol composition, we hypothesized that this strain would be more susceptible to osmotic stresses compared to the $\mathrm{S} 288 \mathrm{C}$ lab strain. This is reasonable since membrane fluidization, governed by ergosterol composition, has been shown to enhance osmotic stress tolerance (Rodríguez-Vargas, Sánchez-García, Martínez-Rivas, Prieto, \& Randez-Gil, 2007). To test this, we performed MICs to compare relative growth across a $\mathrm{NaCl}$ gradient between $\mathrm{S} 288 \mathrm{C}$ and the $E R G 3 \Delta$ strain. 
In our second approach, we selected for fluconazole resistance and used resistant isolates to assess fitness trade-offs. Although several approaches exist to study the presence of trade-offs, consensus exists that strong evidence for trade-offs comes from selection experiments where correlated changes in trait values are measured under directional selection (Bennett \& Lenski, 2007). To test if the previously discussed trade-off exists for strains selected for fluconazole resistance, we allowed for the evolution of fluconazole resistance at a single high concentration of $256 \mu \mathrm{g} / \mathrm{ml}$ fluconazole. Following the selection experiment, two strains, Ev1 and Ev2, were isolated and characterized for fluconazole resistance and $\mathrm{NaCl}$ tolerance relative to their ancestor, S288C, using MIC tests.

\subsubsection{Heat Shock Tolerance-Growth Trade-off}

Yeasts, like all cells, grow and function optimally in a given temperature range. Exposure to temperatures outside this range can result in suboptimal performance or, at extreme temperatures, cell death. However, yeasts can tolerate relatively high temperatures, to some extent, due to rapid, transient and non-heritable physiological changes that ensure cell survival and functioning at elevated temperatures (Piper, 1993). Physiological responses to elevated temperatures begin with the environmental stress response (ESR) which entails the transcription of early stress response genes (Berry \& Gasch, 2008) followed by the heat shock response (HSR) which occurs with a 
change in gene expression and the induction of heat shock proteins (HSPs) and trehalose (Kültz, 2005).

HSPs are a family of proteins, members of which act as molecular chaperones. These proteins operate as part of the HSR to protect cells against moderately elevated temperatures $\left(<45^{\circ} \mathrm{C}\right)$ (Craig, Gambill, \& Nelson, 1993; Sanchez \& Lindquist, 1990). The functions of HSPs include preventing the aggregation of damaged proteins, refolding damaged proteins, and targeting damaged proteins for degradation. Thus, while the HSPs are vital to the heat stress response, the induction of HSPs appear to be a more general response that is also important for protection against a variety of other stresses including UV and oxidative stresses (Verghese, Abrams, Wang, \& Morano, 2012).

The reserve carbohydrate trehalose is a non-reducing disaccharide that protects cells against adverse environmental conditions including desiccation, cold, heat and hypersalinity (Eleutherio, Araujo, \& Panek, 1993; Sano, Asakawa, Inoue, \& Sakurai, 1999; Tapia, Young, Fox, Bertozzi, \& Koshland, 2015). The accumulation of trehalose in response to environmental stressors is an evolutionarily conserved response found in prokaryotes and eukaryotes alike. Within the purview of heat stress response, trehalose primarily functions to protect cells against acute heat stress $\left(>45^{\circ} \mathrm{C}\right)$ (De Virgilio, Hottiger, Dominguez, Boller, \& Wiemken, 1994). Trehalose contributes to thermotolerance by stabilizing proteins, acting as an antidesiccation agent and preventing the aggregation of damaged proteins. While some of the functions of trehalose are similar to those of HSPs, it has been shown that trehalose and Hsp104 (an important protein chaperone from the HSP family) are both necessary forheat shock 
tolerance. This indicates that the functions of HSPs and trehalose do not overlap but rather complement each other (Elliott, Haltiwanger, \& Futcher, 1996).

While the heat stress response induces HSPs and trehalose, it also concomitantly represses protein biosynthesis and is directly associated with a reduction in growth rate at elevated temperatures $\left(>34^{\circ} \mathrm{C}\right)$ (Caspeta et al., 2014). Adaptive laboratory experiments have shown that thermal evolution for prolonged periods of time can overcome this reduction in growth rate at elevated temperatures. However, this evolved thermotolerance comes at the cost of growth at ancestral temperatures (temperature conditions prior to thermal evolution) (Caspeta, Chen, \& Nielsen, 2016; Caspeta \& Nielsen, 2015; Edgardo, Carolina, Manuel, Juanita, \& Baeza, 2008). To evolve thermotolerance and to test for the presence of a trade-off between growth at ancestral temperatures and thermotolerance, we adapted Saccharomyces cerevisiae S288C to elevated temperatures $\left(40^{\circ} \mathrm{C}\right)$ for $\sim 300$ generations. Following this, one strain was isolated ( $\mathrm{T} 1$ ) and was characterized for thermotolerance and growth at ancestral conditions $\left(30^{\circ} \mathrm{C}\right)$. Because thermotolerant cells are also resistant to heat shocks (Wallace-Salinas \& Gorwa-Grauslund, 2013), we used heat shock tolerance as a measure of thermotolerance. Competitive fitness using a reference strain was used as a measure for fitness at $30^{\circ} \mathrm{C}$.

The previously discussed trade-off between thermotolerance and growth at ancestral temperatures has only been observed in the case of adaptation to prolonged 
exposure to elevated temperatures. Because bet hedging is expected to evolve under fluctuating selection even where extreme events are rare, incorporating thermal adaptation in an evolution regime would require the use of transient heat shocks. Here, we ask if thermotolerance that evolved in response to heat shocks also exhibits a tradeoff in fitness at ancestral temperatures. To test this, the isolate from the $40^{\circ} \mathrm{C}$ evolution regime (T1) was further evolved in a regime consisting of frequent $54{ }^{\circ} \mathrm{C}$ heat shocks (once every $\sim 8$ generations) for $\sim 100$ generations. Following this, one strain was isolated (Iso-1) and characterized for heat shock tolerance and relative fitness at $30^{\circ} \mathrm{C}$. We predict that (i) Iso-1 will have evolved greater heat shock tolerance relative to the ancestor, the T1 strain, and (ii) Iso-1 will have lower fitness under benign conditions relative to the ancestor, the T1 strain. This would support the hypothesis that evolved heat shock tolerance comes at the cost of fitness under benign conditions and would provide insight into how this trade-off could be incorporated in fluctuating selection regimes for the evolution of bet hedging.

\subsection{Materials and Methods}

\subsubsection{Strains and culture conditions:}

The yeast strain used for experimental evolution was S288C (MATa SUC2 gal2 mal2 mel flo1 flo8-1 hap1 ho bio1 bio6). The ERG3D strain used to study the trade-off between fluconazole resistance and $\mathrm{NaCl}$ tolerance had a $\mathrm{BY} 4741$ background

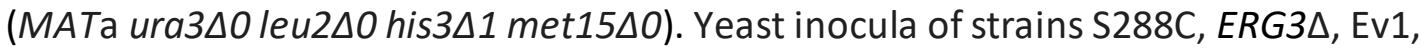


Ev2, T1 and Iso-1 were prepared by growing for $24 \mathrm{~h}$ (for susceptibility testing and competition assays) or $48 \mathrm{~h}$ (for heat shock tolerance assays) in $5 \mathrm{ml}$ Yeast Peptone Dextrose (YPD) broth using a shaker incubator at $30^{\circ} \mathrm{C}$. For susceptibility testing, cultures were then suspended in YPD and cell concentrations were adjusted by spectrophotometry to $1-5 \times 10^{5} \mathrm{cfu} / \mathrm{ml}$. Within 30 minutes of preparation of this working suspension, $100 \mu \mathrm{l}$ was used to inoculate wells 1 to 11 of each row of the microtitration plate to a final cell concentration of $0.5-2.5 \times 10^{5} \mathrm{cfu} / \mathrm{ml}$. After the addition of the inoculum, the final fluconazole concentration range was established at $256 \mu \mathrm{g} / \mathrm{ml}$ to 0.5 $\mu \mathrm{g} / \mathrm{ml}$ and the final $\mathrm{NaCl}$ concentration range was $1 \mathrm{M}$ to $0.002 \mathrm{M}$.

\subsubsection{Preparation of microtitration plates of fluconazole and $\mathrm{NaCl}$ :}

The antifungal agent fluconazole was purchased from Sigma-Adrich and stock solutions were prepared in DMSO at a concentration of $51.2 \mathrm{mg} / \mathrm{ml}$. Working solutions were prepared by 100 -fold dilutions of stock solution in YPD. Sterile 96 -well flat-bottom microtitration plates were used to make two-fold dilutions of the drug spanning a concentration range of $512 \mu \mathrm{g} / \mathrm{ml}$ to $1 \mu \mathrm{g} / \mathrm{ml}$. Columns 1 to 10 of the microtitration plate contained $100 \mu \mathrm{l}$ of Fluconazole + YPD. Column 11 was an agent-free control with $100 \mu \mathrm{l}$ of YPD and column 12 was a media control used for background $\mathrm{OD}_{600}$ correction. The prepared microtitration plates can be stored for $<1$ week at $-20^{\circ} \mathrm{C}$. 
$\mathrm{NaCl}$, purchased from BioShop, was prepared at concentrations of $2 \mathrm{M}$ in sterile $\mathrm{dH}_{2} \mathrm{O}$. Following a similar protocol, a concentration range spanning $1 \mathrm{M}$ to $0.004 \mathrm{M}$ was prepared on microtitration plates.

\subsubsection{Trait assays:}

Minimum inhibitory concentration test

The microtitration test plates were incubated with shaking at $30{ }^{\circ} \mathrm{C}$ for $48 \mathrm{~h}$ and analyzed using a plate-reader (Cytation 5, BioTek Instruments). OD 600 measurements were taken every 30 minutes. Growth was recorded across the concentration gradient and normalized to the agent-free control well. The normalized data was used to identify MIC values which was defined as the smallest concentration at which $50 \%$ growth inhibition occurs relative to the agent-free control. If $>50 \%$ growth inhibition did not occur across the entire tested concentration gradient, the breakpoint was arbitrarily recorded as one step above the highest concentration.

\section{$\underline{\text { Heat Shock Tolerance assays }}$}

As estimates of heat shock tolerance are only consistent at late saturation phase of growth (Appendix A), heat shock tolerance was assayed at 48 hours after incubation. Cells were inoculated and allowed to grow in Synthetic Defined Media (SDM) at $30^{\circ} \mathrm{C}$. At $48 \mathrm{~h}$ post incubation, cells were resuspended in SDM and adjusted by 
spectrophotometry to an optical density at $600 \mathrm{~nm}\left(\mathrm{OD}_{600}\right)$ of $0.05 .250 \mu \mathrm{L}$ of this cell suspension was pipetted into microcentrifuge tubes in duplicate and heat shocked at 54 ${ }^{\circ} \mathrm{C}$ for 30 minutes. Following the heat shock, the microcentrifuge tubes were kept on ice until plating within $30 \mathrm{~min}$. Both heat shocked and non-heat shocked cell suspensions were spread on YPDA to estimate viable cell counts. Heat shock tolerance was measured as a percentage of surviving cells relative to the total number of cells.

\section{Competitive fitness assays}

Relative fitness under benign conditions $\left(30^{\circ} \mathrm{C}\right)$ was measured as competitive fitness using a reference strain. The reference strain used, obtained from the yeast nonessential gene deletion array, had a BY4741 background (MATa ura3 $\Delta 0$ leu2 $\Delta 0$ his $3 \Delta 1$ met15 $\Delta 0$ ) and was YIR044C $\Delta$. YIR044C is a pseudogene and is replaced by a gene conferring resistance to the drug G418 (Sigma-Aldrich). G418-resistance was used as a marker for subsequent competitive fitness assays as the focal strains were all susceptible to G418. All focal strains and the reference strain were grown to saturation in YPD at $30{ }^{\circ} \mathrm{C}$ and resuspended using spectrophotometry to an $\mathrm{OD}_{600}$ of 0.05 . Following the resuspension, the reference strain and individual focal strains were competed in YPD for 48 hours. Proportions of focal strain relative to the reference strain both before and after the competition were determined by plating on YPDA and YPDA+G418 (G418 concentration of $200 \mu \mathrm{g} / \mathrm{ml}$ ). Relative fitness was calculated according to the protocol described in Wong, Rodrigue \& Kassen (2012) where the 
selection coefficient was determined using the equation described below. Fitness $w$ was calculated as $1+s$.

$$
s=\frac{\ln \frac{\text { focalinitial }}{\text { focalfinal }}-\ln \frac{\text { referenceinitial }}{\text { referencefinal }}}{\text { No. of generations }}
$$

\subsubsection{Experimental evolution:}

Three different experimental evolution experiments were conducted in this study. In the first experimental evolution regime, fluconazole resistance was evolved using $\mathrm{S} 288 \mathrm{C}$ as the progenitor following the protocol adapted from Anderson et. al, 2003. Cells from an independent colony were grown overnight and a cell suspension having a concentration of $5 \times 10^{4} \mathrm{cfu} / \mathrm{ml}$ was prepared and spread in duplicate onto solid YPD media containing $256 \mu \mathrm{g} / \mathrm{ml}$ of fluconazole. After 4 days of incubation at $30^{\circ} \mathrm{C}$, the largest, continuously growing colony from each plate was picked and assayed for fluconazole resistance by MIC methods.

In the second evolution regime, thermotolerance was evolved using S288C as the progenitor following a protocol adapted from Caspeta et al. (2014). Cells from an individual colony were grown in YPD for 24 hours at $40 \pm 0.3^{\circ} \mathrm{C}$ and diluted into fresh medium to an $\mathrm{OD}_{600}$ of 0.1 . This was repeated for 36 days ( 300 generations) following which a cell suspension was prepared and plated on solid YPDA. The calculations for the number of generations is described in Appendix C. One colony was isolated (T1) and characterized for heat shock tolerance and fitness under benign conditions $\left(30^{\circ} \mathrm{C}\right)$. 
In the third evolution regime, heat shock tolerance was evolved using the T1 isolate as the progenitor. Cells from an individual colony were grown in YPD for 48 hours following which a cell suspension was prepared by dilution to an $\mathrm{OD}_{600}$ of $0.05 .250 \mu \mathrm{L}$ of this cell suspension was pipetted into microcentrifuge tubes and heat shocked at $54^{\circ} \mathrm{C}$ for 30 minutes. $100 \mu \mathrm{L}$ of the heat shocked suspension was used to inoculate $5 \mathrm{ml}$ of fresh media. Because heat shocked cells take longer to resume growth, subsequent heat shocks were applied 72 hours after heat shocked cells were inoculated in fresh media. This was repeated for 36 days (13 heat shocks, $\sim 100$ generations). One colony was isolated (Iso-1) and characterized for heat shock tolerance and growth under benign conditions $\left(30^{\circ} \mathrm{C}\right)$.

\section{$\underline{1.2 .5}$ Statistical analyses:}

Analyses of fluconazole and $\mathrm{NaCl}$ MICs for the four strains S288C, ERG3D, Ev1 and Ev2 were performed using one-way ANOVA tests. Five independent population measurements from each strain were used for statistical testing of fluconazole and $\mathrm{NaCl}$ MIC endpoints. MIC endpoints were $\log _{2}$ transformed prior to statistical analysis to account for fold changes in concentration. Following the one- way ANOVA tests, posthoc Tukey tests were performed to make pair-wise comparisons of MICs across strains.

Analysis of heat shock tolerance and competitive fitness for the three strains S288C, T1 and Iso-1 were performed using one-way ANOVA tests. Six trait measurements from independent populations of tested strains were used to statistically 
compare heat shock tolerance. Three trait measurements from independent populations of tested strains were used to statistically compare competitive fitness.. Post-hoc Tukey tests were performed to make pair-wise comparisons of heat shock tolerance and competitive fitness across strains. Statistical packages used were $R$ and Prism Graphpad.

\subsection{Results}

\subsubsection{Fluconazole resistance $-\mathrm{NaCl}$ tolerancetrade-off}

One-way ANOVAs performed for fluconazole and NaCl MICs (Tables 1.1, 1.2) show that both fluconazole MIC endpoints and $\mathrm{NaCl}$ MIC endpoints significantly differed across strains ( $p<0.0001$ for both tests). A post-hoc Tukey test for fluconazole MICs (Table 1.3) revealed that all strains significantly differed from each other $(p<0.001)$ except for $E R G 3 \Delta$ and Ev2 which had similar fluconazole MICs ( $p>0.999)$. Similarly, a post-hoc Tukey test for $\mathrm{NaCl}$ MICs (Table 1.4) showed that all pairwise comparisons of strains were significantly different $(p<0.001)$ except for the Ev1-S288C $(p=0.31)$ and Ev1-ERG3D pairs $(\mathrm{p}=0.07)$. 
The ERG3 $\Delta$ strain had the greatest resistance to fluconazole with a MIC of 512 $\mu \mathrm{g} / \mathrm{ml}$ and the lowest $\mathrm{NaCl}$ tolerance with a MIC of $0.5 \mathrm{M}$. S288C had the lowest resistance to fluconazole with a MIC of $64 \mu \mathrm{g} / \mathrm{ml}$ and an intermediate $\mathrm{NaCl}$ tolerance with a MIC of $1 \mathrm{M}$. The isolate Ev1 was intermediate both in terms of fluconazole resistance and $\mathrm{NaCl}$ tolerance with MICs of $256 \mu \mathrm{g} / \mathrm{ml}$ and $1 \mathrm{M}$ respectively. The isolate Ev2 had both the greatest resistance to fluconazole and greatest tolerance to $\mathrm{NaCl}$ with MICs of $512 \mu \mathrm{g} / \mathrm{ml}$ and $2 \mathrm{M}$ respectively. The highest MIC concentration values reported here $(512 \mu \mathrm{g} / \mathrm{ml}$ and $2 \mathrm{M})$ are each one concentration step above the actual range tested and were reported when $50 \%$ growth inhibition did not occur even at the highest concentration $(256 \mu \mathrm{g} / \mathrm{ml}$ or $1 \mathrm{M})$.

\subsection{2 $\underline{\text { Heat shock tolerance - Growth trade-off }}$}

A one-way ANOVA test (Table 1.5) showed that heat shock tolerance significantly differed across strains $(p<0.0001)$. A follow up post-hoc Tukey test revealed that heat shock tolerances for strains were significantly different from each other (Table 1.6). S288C, the progenitor of all other thermotolerant strains, had the lowest heat shock tolerance (Mean $=5.17 \%)$. The T1 strain, isolated from a population evolved at elevated temperatures $\left(40^{\circ} \mathrm{C}\right)$, had a mean heat shock tolerance of $21.23 \%$. The isolate from the heat shock regime, Iso-1, had a heat shock tolerance of $59.91 \%$. Fitness under benign conditions, analyzed using a one-way ANOVA (Table 1.7), also significantly differed across strains $(p<0.0001)$. A post-hoc Tukey test revealed that all strains significantly 
differed from each other (Table 1.8). Surprisingly, the progenitor S288C had the lowest competitive fitness with a mean relative fitness of 0.942 . The T1 strain, evolved from S288C at elevated temperatures, had the highest competitive fitness with a mean relative fitness of 1.156 . The isolate from the heat shock regime, Iso-1, had an intermediate fitness under benign conditions with a mean competitive fitness of 0.987 . These results indicate that while evolution to elevated temperatures $\left(40^{\circ} \mathrm{C}\right)$ resulted in an increase in heat shock tolerance relative to the ancestor $\mathrm{S} 288 \mathrm{C}(\mathrm{p}=0.0047)$, it did not come at the cost of relative fitness. Rather, competitive fitness was found to be significantly higher for the T1 strain compared to S288C $(p<0.001)$. On the other hand, while evolution under a regime of heat shocks $\left(54{ }^{\circ} \mathrm{C}\right.$ for $\left.30 \mathrm{mins}\right)$ resulted in an increase in heat shock tolerance relative to the ancestral T1 strain $(p<0.001)$, it also resulted in a significant decrease in competitive fitness $(p<0.001)$. 
A

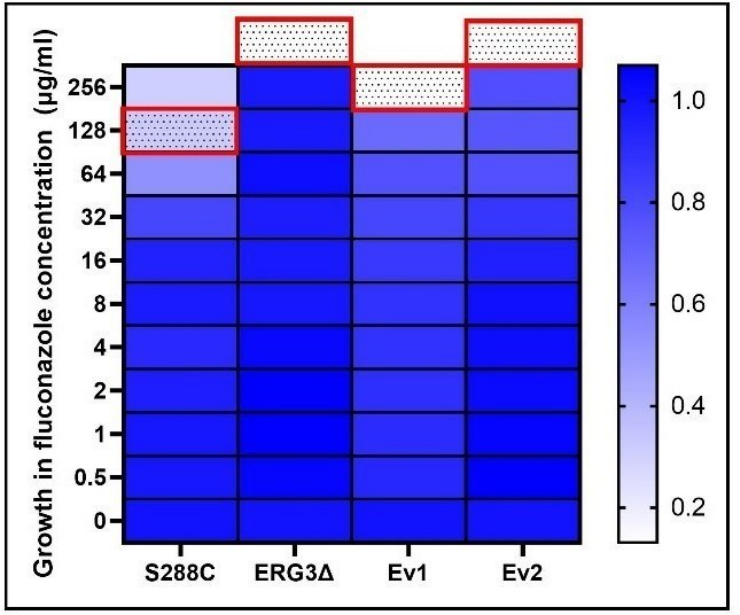

B

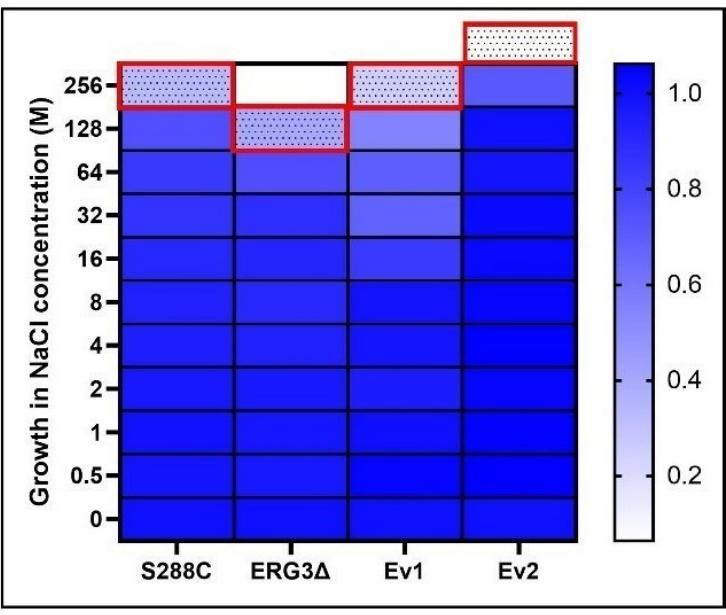

Figure 1.1: Relative growth of S288C, ERG3A, Ev1 and Ev2 strains across (A) fluconazole and (B) $\mathrm{NaCl}$ gradients. Red boxes denote MIC values for each strain (placed above highest concentration tested if growth inhibition does not occur across entire range). Yield values across concentration gradient are relative to agent-free controls (bottom cells). Gradient bar on the right of each heat map represents normalized $\mathrm{OD}_{600}$.

A

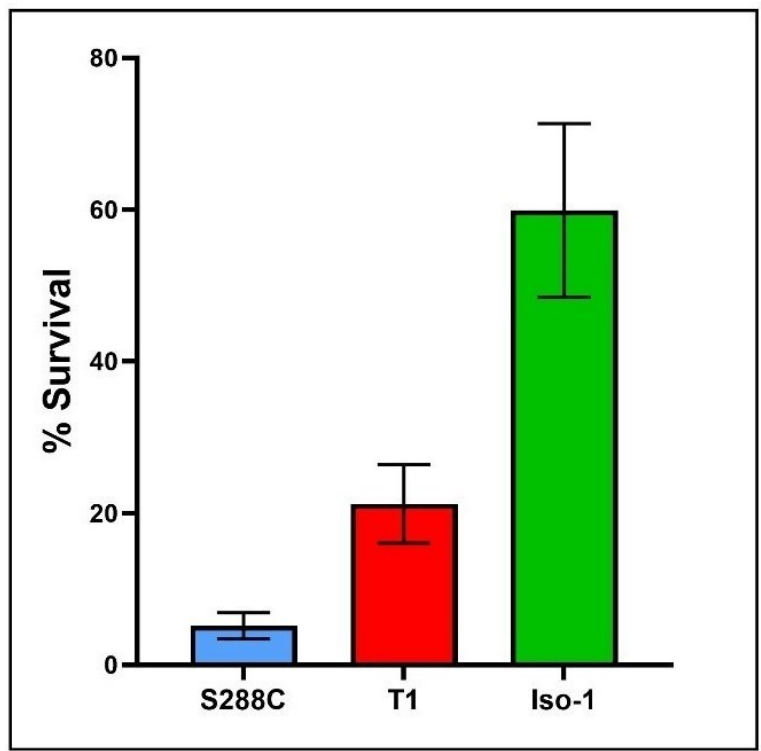

B

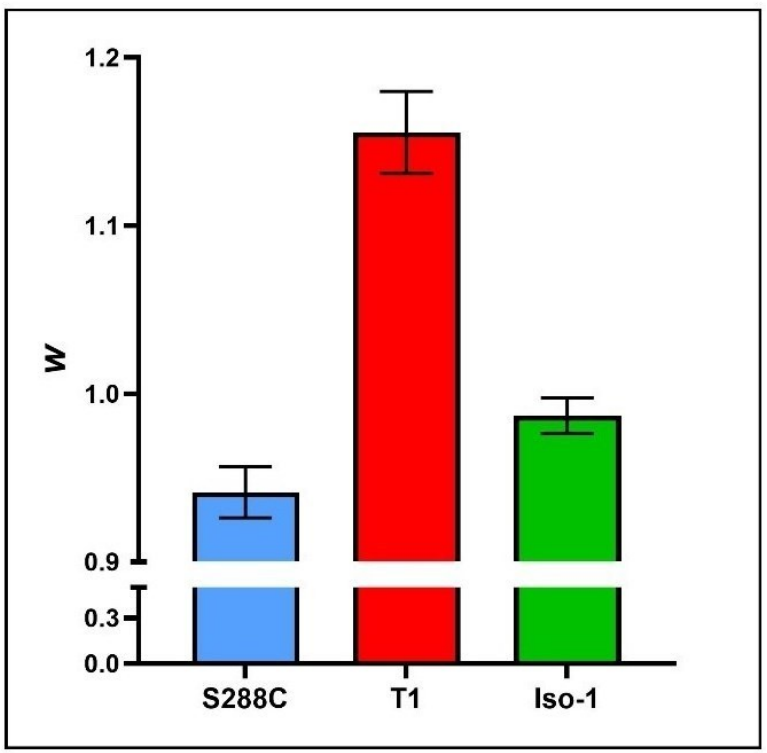

Figure 1.2: (A) Heat shock tolerance (measured as \% Survival) and (B) competitive fitness ( $w$ ) of S288C, T1 and Iso-1, relative to YIR044CA. Error bars represent SD. 
Table 1.1: Results of a One-Way ANOVA for differences in fluconazole MICs across strains. The strains compared were S288C, ERG3D, Ev1 and Ev2.

\begin{tabular}{lcccc}
\hline \multicolumn{1}{c}{ Source } & $d f$ & $M S$ & $F$ & $p$ \\
\hline Between groups & 3 & 7.533 & 100.4 & $<.0001$ \\
Within groups & 16 & 0.075 & & \\
Total & 19 & & & \\
\hline
\end{tabular}

Table 1.2: Results of a One-Way ANOVA for differences in NaCl MICs across strains. The strains compared were S288C, ERG3

\begin{tabular}{|c|c|c|c|c|}
\hline Source & $d f$ & $M S$ & $F$ & $p$ \\
\hline Between groups & 3 & 2.850 & 22.80 & $<.0001$ \\
\hline Within groups & 16 & 0.125 & & \\
\hline Total & 19 & & & \\
\hline \multicolumn{5}{|c|}{$\begin{array}{l}\text { Table 1.3: Post-hoc Tukey test results for differences in fluconazole MICs across strains. Common lette } \\
\text { indicate homogenous subsets. Letters that are not shared indicate significantly different mean values. }\end{array}$} \\
\hline Group & Lette & $\begin{array}{l}\text { ig homog } \\
\text { sets }\end{array}$ & & Modal MIC \\
\hline S288C & A & & & $64 \mu \mathrm{g} / \mathrm{ml}$ \\
\hline ERG3 $\triangle$ & & B & & $512 \mu \mathrm{g} / \mathrm{ml}$ \\
\hline Ev2 & & B & & $512 \mu \mathrm{g} / \mathrm{ml}$ \\
\hline Ev1 & & & & $256 \mu \mathrm{g} / \mathrm{ml}$ \\
\hline
\end{tabular}


Table 1.4: Post-hoc Tukey test results for differences in NaCl MICs across strains. Common letters indicate homogenous subsets. Letters that are not shared indicate significantly different mean values.

\begin{tabular}{lccr}
\hline Group & \multicolumn{2}{c}{$\begin{array}{c}\text { Letters showing homogenous } \\
\text { subsets }\end{array}$} & Modal MIC \\
S288C & A & $1 \mathrm{M}$ \\
Ev1 & A & B & $1 \mathrm{M}$ \\
ERG3 & B & C & $0.5 \mathrm{M}$ \\
Ev2 & & $2 \mathrm{M}$ \\
\hline
\end{tabular}

Table 1.5: Results of a One-Way ANOVA for differences in heat shock tolerance across strains. The strains compared were S288C, T1 and Iso-1.

\begin{tabular}{lcccc}
\hline \multicolumn{1}{c}{ Source } & $d f$ & $M S$ & $F$ & $p$ \\
\hline Between groups & 2 & 4751 & 88.58 & $<.0001$ \\
Within groups & 15 & 53.64 & \\
Total & 17 & & \\
\hline
\end{tabular}


Table 1.6: Post-hoc Tukey test results for differences in heat shock tolerance across strains. Common letters indicate homogenous subsets. Letters that are not shared indicate significantly different mean values.

\begin{tabular}{lccc}
\hline Group & \multicolumn{2}{c}{ Letters showing homogenous } & Mean HST \\
S288s C & A & B & 5.17 \\
T1 & & C & 21.23 \\
Iso-1 & & C & 59.91 \\
\hline
\end{tabular}

Table 1.7: Results of a One-Way ANOVA for differences competitive fitness across strains. The strains compared were S288C, T1 and Iso-1.

\begin{tabular}{lcccc}
\hline \multicolumn{1}{c}{ Source } & $d f$ & $M S$ & $F$ & $p$ \\
\hline Between groups & 2 & 0.03815 & 121.8 & $<.0001$ \\
Within groups & 6 & 0.0003133 & & \\
Total & 8 & & & \\
\hline
\end{tabular}

Table 1.8: Post-hoc Tukey test results for differences in competitive fitness across strains. Common letters indicate homogenous subsets. Letters that are not shared indicate significantly different mean values.

\begin{tabular}{lccc}
\hline Group & \multicolumn{2}{c}{$\begin{array}{c}\text { Letters showing homogenous } \\
\text { subsets }\end{array}$} & Mean fitness \\
\hline S288C & A & B & 0.942 \\
T1 & & C & 1.156 \\
Iso-1 & & C & 0.987 \\
\hline
\end{tabular}




\subsection{Discussion}

Fitness trade-offs cause variance in fitness across environments and, because of this, are central to the evolution of bet hedging. The objective of this study was to assess two different systems for the presence of a trade-off using an experimental evolution approach. In the first system, that tested the trade-off between fluconazole resistance and salt tolerance, a trade-off was observed on comparing the $E R G 3 \Delta$ strain (obtained from the non-essential gene deletion array) to the lab strain S288C. Here,MIC tests showed that $E R G 3 \Delta$ had a higher resistance to fluconazole and a lower tolerance to $\mathrm{NaCl}$ relative to $\mathrm{S} 288 \mathrm{C}$. This increased resistance to fluconazole and decreased tolerance to $\mathrm{NaCl}$ in the $E R G 3 \triangle$ strain is in keeping with results obtained from largescale gene deletion phenotype studies (Dudley, Janse, Tanay, Shamir, \& Church, 2005; Yoshikawa et al., 2011). The altered sterol composition causing hyperpolarization of the cell membrane in the ERG3 $\Delta$ strain has been shown to mediate this trade-off (Kodedová \& Sychrová, 2015). However, the fluconazole resistant isolates (Ev1 and Ev2) obtained from the selection experiment did not show this trade-off. Fluconazole resistance evolved by subjecting populations to a single high dose of fluconazole, as in this study, has been shown to result in recessive mutations in ERG3 causing the overexpression of ERG11 which encodes the drug target. Ev1 had a significantly higher fluconazole resistance relative to the ancestor S288C. However, neither Ev1 nor the ancestor differed significantly in terms of tolerance to $\mathrm{NaCl}$, suggesting that fluconazole resistance did not evolve at the cost of $\mathrm{NaCl}$ tolerance. A similar observation was made 
in the case of Candida albicans where fluconazole resistant and sensitive strains did not differ in their response to osmotic stress (Kolecka, Krauke, Bujdakova, \& Sychrova, 2009). In the present study, the isolate Ev2 had the highest fluconazole resistance amongst all strains that were compared, including the ancestor S288C. Surprisingly, Ev2 also had the highest tolerance to $\mathrm{NaCl}$ compared to all other strains. This implies a possible mechanism where resistance to fluconazole also confers cross-resistance to $\mathrm{NaCl}$. The difference in trade-offs observed between the isolates Ev1 and Ev2 is potentially indicative of different mechanisms underlying evolved fluconazole resistance that may each come with varying costs. In addition, studies have shown that even if the evolution of drug resistance does come at a fitness cost initially, these costs can be eliminated by further evolution though compensatory mutations (Levin, Perrot, \& Walker, 2000; Maisnier-Patin \& Andersson, 2004; Zhang, Sahin, McDermott, \& Payot, 2006). The elimination of fitness costs has also been observed in the case of evolved fluconazole resistance where an initial fitness cost was alleviated though further evolution without affecting fluconazole resistance (Cowen, Kohn, \& Anderson, 2001).

A system developed for the study of bet hedging requires a reliable, or "hard" evolutionary trade-off. In this system, while a trade-off was observed in the ERG3A strain, it was not observed in the case of evolved fluconazole resistance. Therefore, if this system was used to impose fluctuating selection, the evolutionary strategy that would maximize the GMF would not be bet hedging but rather the independent maximization of both fluconazole resistance and $\mathrm{NaCl}$ tolerance. However, while a 
trade-off was not observed in this study, it is difficult to rule out costs of adaptation to fluconazole entirely. It is possible that trade-offs associated with fluconazole resistance are manifested in alternative environments that have not been tested in this study. Future studies on trade-offs associated with fluconazole resistance should explore fitness costs using a variety of stressors and media conditions.

In the second system, the trade-off between thermotolerance and growth at ancestral temperatures was assessed. Thermotolerance evolution in yeast is associated with a reduction in growth-related cellular functions which, while critical to the acquisition of thermotolerance, is also correlated with decreased fitness at ancestral temperatures (temperature conditions prior to thermal evolution) (Zeldovich, Chen, \& Shakhnovich, 2007). This trade-off in fitness at ancestral temperatures has also been observed in a variety of systems including of viruses, bacteria and ectotherms (Bennett \& Lenski, 2007; Huey \& Kingsolver, 1989; Knies, Izem, Supler, Kingsolver, \& Burch, 2006; White, DeBach, \& Garber, 1970). We found that while evolution at $40{ }^{\circ} \mathrm{C}$ resulted in an increase in heat shock tolerance relative to the ancestor, it did not come at a cost of fitness in ancestral temperatures. Instead, the thermotolerant strain T1 in addition to having higher heat shock tolerance also had higher fitness at $30^{\circ} \mathrm{C}$ relative to the ancestor $\mathrm{S} 288 \mathrm{C}$. This could be due to cross-adaptation where mutations that increase fitness under elevated temperatures also increase fitness under benign conditions. This explanation seems unlikely as numerous studies point to the central trade-off between growth rate and stress-tolerance functions, suggesting that these traits cannot be 
improved independently (Spor, Wang, Dillmann, de Vienne, \& Sicard, 2008; Zakrzewska et al., 2011).

Another possibility is that yeast adapt to rich media conditions simultaneously with thermal evolution. This is characteristic of a multi-trait trade-off in which two or more functions compete for a common limiting resource. An inverse relationship between growth and stress tolerance is a result of fine-tuned physiological pathways that have evolved in both naturally and artificially selected yeast (Deutscher, Meilijson, Kupiec, \& Ruppin, 2006; Vilaprinyo, Alves, \& Sorribas, 2006). However, the pathways that underlie the functions of growth rate and stress tolerance are not entirely independent of each other, sharing partial overlap, which can result in the simultaneous, albeit limited, maximization of both functions (Castrillo et al., 2007; S. Levy et al., 2007). This is likely the case in the heat shock tolerance - growth trade-off system, as yeast in this study were evolved in nutrient rich medium (YPD) in which resources are abundant. Under such conditions, a trade-off is not manifested until the shared resource becomes limiting. If resource limitation does underlie this trade-off, further adaptation to heat should result in the loss of fitness under benign conditions. Indeed, we find that resource limitation does underlie this trade-off as further evolution experiments using a regime consisting of frequent heat shocks resulted in a fitness cost of adaptation in the isolate Iso-1 relative to the T1 ancestor. Taken together, these results indicate the existence of a multi-trait trade-off between heat shock tolerance and growth under benign conditions mediated by competition for a limiting resource. In 
further support for this trade-off, evolving heat shock tolerant strains under benign conditions (Appendix B) resulted in an increase in competitive fitness under benign conditions that come at the cost of heat shock tolerance. Because the trade-off is only manifested after both traits are improved to a degree, we suggest the use of the T1 isolate for thermal evolution regimes to evolve bet hedging. While this strain has both a higher heat shock tolerance and fitness under benign conditions relative to the ancestor, further improvement in heat shock tolerance comes at a cost of fitness under benign conditions. We also suggest the use of nutritionally poor media for the evolution of yeast cultures to accentuate the effects of resource limitation that underlie this trade-off. This study demonstrates that evolved strains gain heat shock tolerance at the cost of fitness in benign environments, suggesting the presence of a trade-off. To better understand how phenotypic differences in heat shock tolerance and fitness in benign conditions are manifested and if quantitative relationships in this trade-off exist, future studies should explore this trade-off on genetic, transcriptomic and proteomic levels. 


\section{Chapter 2: Bet hedging evolves as a constraint on adaptation}

\subsection{Introduction}

The natural environment is a composite of diverse environmental factors that are each in a constant state of flux. These environmental factors vary both spatially and temporally and cause dynamic changes in fitness. The resulting variation in fitness can be quite drastic and can be seen as a factor influencing extinction risk (Balmford, 1996; Vincenzi, 2014). Bet hedging, as a mode of evolutionary response, is a risk-aversion strategy that allows organisms to persist in the face of these fluctuations (Lewontin \& Cohen, 1969; Philippi \& Seger, 1989). A bet-hedging strategy maximizes long-term, or geometric mean, fitness through a reduction in fitness variance across environments by sacrificing fitness in average or expected environments in exchange for increased fitness in rare but calamitous environments.

The surge of interest in bet hedging in recent years (Frankenhuis, Panchanathan, \& Belsky, 2016; Furness, Lee, \& Reznick, 2015; García-Roger, Serra, \& Carmona, 2014; Graham, Smith \& Simons, 2014; Gremer, Kimball, \& Venable, 2016; Lycus et al., 2018; Tarazona, García-Roger \& Carmona, 2017) has led to a comprehensive understanding of the prevalence and circumstances under which bet hedging evolves. Despite the extensive theoretical groundwork for bet hedging and growing empirical evidence that it exists, how bet hedging evolves remains an unanswered question. The process that 
maximizes long- 
term fitness necessarily entails the selective maintenance of suboptimality over shorter timescales. This can only occur through selection for the ability to not strongly respond to selection in the short-term.

The idea that bet hedging evolves as a constraint on adaptive tracking has been proposed as a conceptual model (Simons, 2002) but it has been neither mathematically modelled nor empirically tested. Adaptation is not instantaneous and always lags behind a change in the environment. This limitation on the course of adaptation which impedes the outcome of adaptive tracking is an evolutionary constraint. Different types of evolutionary constraints exist including i) Genetic constraints (limitation on standing genetic variation), ii) Developmental constraints (limitation on developmental states that can be expressed) and, iii) Functional constraints (limitation on trait values or a combination of trait values)(Arnold, 1992; Blows \& Hoffmann, 2005). The study of constraints has focused on their source, strength, immediate consequence and persistence, and constraints have traditionally been viewed as hinderances to adaptive evolution. In this study, we propose to empirically test the idea that in the face of fluctuating selection, constraints can be adaptive when they are evolved as a bethedging trait. If genetic variation in constraint exists, selection can act on this variation. In doing so, fitness in rare but detrimental environments is not compromised and a constraint on adaptive tracking increases long-term, or geometric-mean fitness.

Bet hedging is expected to evolve when fitness values fluctuate across environments due to fitness trade-offs. Trade-offs exist when traits have opposing effects on fitness (Garland, 2014). In the absence of trade-offs, traitscan evolve 
independently; thus, evolution of phenotypes that are well adapted to all environments would be expected. However, if trade-offs prevent simultaneous adaptive evolution of both traits, the evolution of bet hedging mitigates fluctuations in fitness values. This study takes an experimental evolution approach in the yeast Saccharomyces cerevisiae and, using a Heat Shock Tolerance-Growth trade-off system (as described in Chapter 2, Section 2.3), we test the hypothesis that bet hedging evolves as a constraint on adaptive tracking. To ask if a constraint evolves under environmental fluctuation, we select for the retention of the bet-hedging trait (heat shock tolerance) through a reduction in the frequency of extreme environments (heat shocks) across two sequential regimes. The two evolution regimes, Regime-A (high frequency of extreme environments) and Regime-B (low frequency of extreme environments), both impose fluctuating selection using heat shocks $\left(54^{\circ} \mathrm{C}\right)$ and benign environments $\left(30^{\circ} \mathrm{C}\right)$. As the frequency of heat shocks decreases from Regime-A to Regime-B, benign conditions become more frequent resulting in increased selective pressure to enhance growth at $30^{\circ} \mathrm{C}$. However, any improvement in growth results in the loss of heat-shock tolerance which is necessary for survival. Therefore, we predict that 'low frequency of extreme environments' within this experimental design selects for a constraint on adaptation to benign environments and, by extension, the retention of heat shock tolerance. Because adaptation is never completely unconstrained and some level of resistance to adaptation exists prior to any variable selection, we begin with a high frequency regime and test for a change in constraint when the frequency of heat shocksdecreases. 
Using the T1 thermotolerant strain (Chapter 2, Section 2.3.1) as the ancestor, 8 replicate lines were first passed through Regime-A for 100 generations and then transferred to Regime-B for 100 generations. We predict the evolution of increased heat shock tolerance and a concomitant decrease in fitness under benign conditions at the end of both Regime-A (EoR-A) and at the end of Regime-B (EoR-B) relative to the ancestor. Testing the hypothesis that bet hedging evolves as a constraint on adaptive tracking requires phenotypic measurements of evolved constraints (as described in Appendix B). Therefore, the test of this hypothesis - that evolution is constrained requires an evolutionary rather than a single time-point assay: we compare evolved constraints at EoR-A and EoR-B by allowing for further evolution to gauge the loss of heat-shock tolerance under constant benign environments, following which both heat shock tolerance and fitness under benign conditions are tested.

Four possible scenarios exist as the outcome for tests of evolved constraints as lines from the two regimes are evolved under benign conditions (Fig. 3.1). In the first scenario (Fig. 3.1.A), a constraint on adaptive tracking does not evolve at EoR-B relative to EoR-A. This is the null expectation and is characterized by lines from EoR-A and EoR-B being indistinguishable in terms of constrained evolution. In the second scenario (Fig. 3.1.B), a constraint on adaptive tracking does not evolve but lines from EoR-A have a higher heat shock tolerance than those from EoR-B both before and after evolution under benign conditions. In this scenario, although lines from EoR-A have a higher heat shock tolerance after evolution under benign conditions, this cannot be attributed to an evolved constraint but rather simply due a higher initial heat shock tolerance compared 
to lines from EoR-B. Similarly, interpretations of evolved constraints can only be made if lines from EoR-B do not have a higher initial heat shock tolerance relative to lines from EoR-A. In the third and fourth scenarios (3.1.C and 3.1.D respectively), lines from EoR-B are more constrained when evolving under benign conditions relative to those from EoR-A. This is characterized by lines from EoR-A losing heat shock tolerance more rapidly than lines from EoR-B after further evolution under benign conditions. In the third scenario, the initial heat shock tolerance of lines from both regimes are indistinguishable whereas in the fourth scenario the initial heat shock tolerance of lines from EoR-B is lower relative to that of lines from EoR-A. This reduction in heat shock tolerance can be attributed to increased selective pressure in Regime-B to improve fitness under benign conditions which is expected to come at a cost of heat shock tolerance. The third and fourth scenarios represent the evolution of a bet-hedging trait in Regime-B, characterized by the retention of heat shock tolerance, that has evolved as a constraint on adaptive tracking. 
A

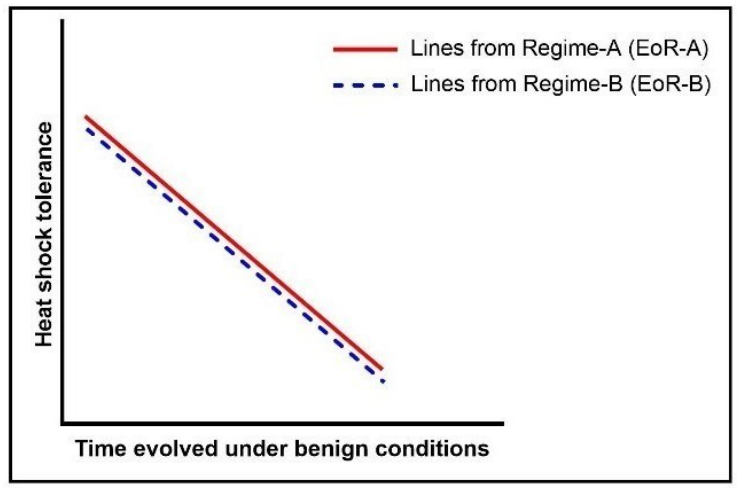

C

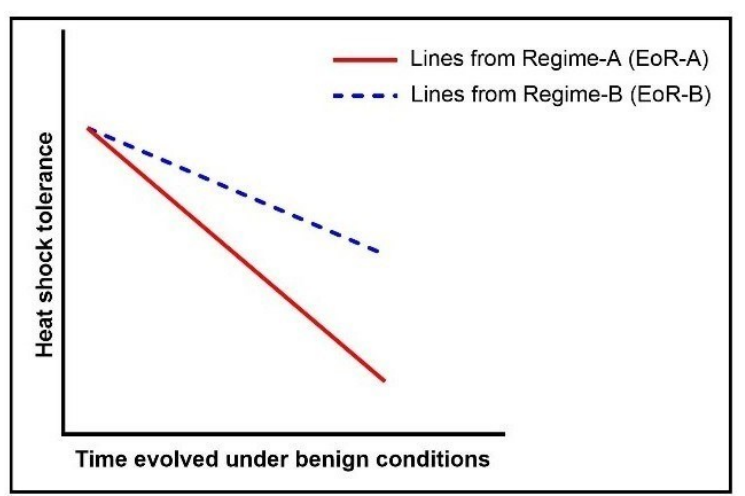

B

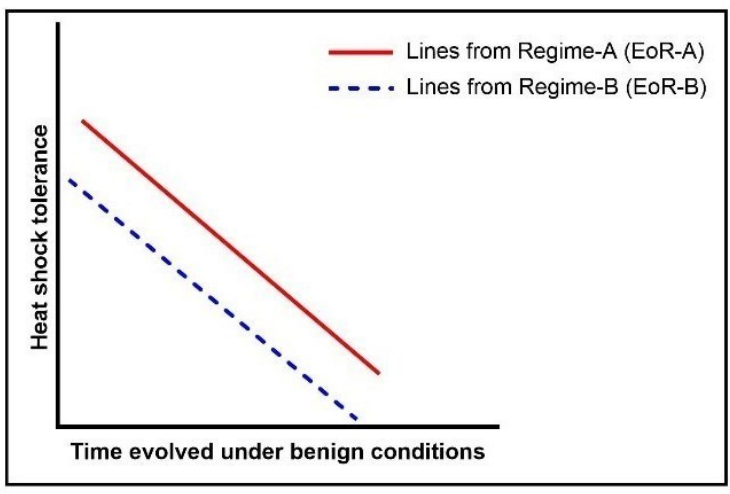

D

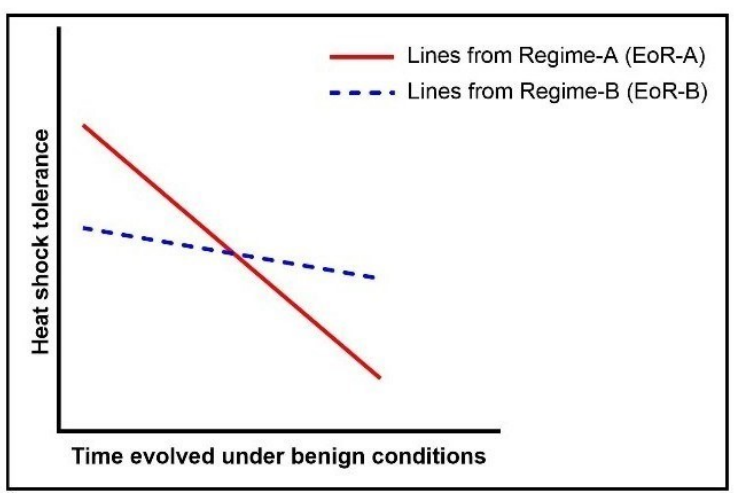

Figure 2. 1: Four possible scenarios representing the outcome of assays of evolved constraints in lines from Regime-B (blue; low-frequency extreme events) relative to those from Regime-A (red; highfrequency extreme events). Scenarios $A$ and $B$ represent the null prediction where a constraint does not evolve in Regime-B relative to Regime-A, i.e., heat shock tolerance is lost at similar rates. Scenarios C and $D$ represent the evolution of constraint in lines from Regime-B relative to lines from Regime-A. This is characterized by the lower evolutionary rate of loss of heat shock tolerance in Regime-B lines relative to Regime-A lines. 


\subsection{Materials and Methods:}

\subsubsection{Strains and culture conditions:}

The previously evolved thermotolerant yeast strain T1 (Chapter 1, Section 1.1.3) with an S288C background (MATa SUC2 gal2 mal2 mel flo1 flo8-1 hap1 ho bio1 bio6) was used as the ancestor for experimental evolution. Yeast inocula using the T1 strain or test populations were prepared by growing for $24 \mathrm{~h}$ (for competition assays) or $48 \mathrm{~h}$ (for heat shock tolerance assays) in $5 \mathrm{ml}$ Synthetic Defined Medium (SDM) broth using a shaker incubator at $30^{\circ} \mathrm{C}$. SDM contains $6.7 \mathrm{~g}$ of Yeast Nitrogen Base (without amino acids with ammonium sulfate) and $2 \%$ dextrose per litre. For competition experiments the reference strain used was YIR044C $\Delta$. YIR044C $\Delta$ was obtained from the yeast nonessential gene deletion array and has a BY4741 background (MATa ura3 $\triangle 0$ leu2 $\triangle 0$ his3 $\Delta 1$ met15 $\Delta 0$ ) and is deleted for the gene YIR044C, a pseudogene that was replaced by a gene conferring resistance to the drug G418. For competition experiments all focal strains, test populations and the reference strain were grown to saturation in SDM+ His (10 mg/L), Met (10 mg/L), Leu (30 mg/L), Ura (10 mg/L). Strains were archived every 20 generations during experimental evolution at $-80^{\circ} \mathrm{C}$ in $15 \%$ glycerol. 


\subsubsection{Trait assays:}

\section{Heat Shock Tolerance assays}

Cells from an individual colony (in the case of the T1 strain) or from a population aliquot (in the case of test populations) were each separately inoculated and allowed to grow in SDM at $30{ }^{\circ} \mathrm{C}$. At $48 \mathrm{~h}$ post incubation, cells were resuspended in SDM and adjusted by spectrophotometry to an optical density at $600 \mathrm{~nm}\left(\mathrm{OD}_{600}\right)$ of $0.05 .250 \mu \mathrm{L}$ of this cell suspension was pipetted into microcentrifuge tubes in duplicate and heat shocked at $54{ }^{\circ} \mathrm{C}$ for 75 minutes. Following the heat shock, the microcentrifuge tubes were kept on ice until plating within $30 \mathrm{~min}$. Both heat shocked and non-heat shocked cell suspensions were spread on YPDA to estimate viable cell counts. Heat shock tolerance was measured as a percentage of cells surviving heat shock relative to the total number of viable cells prior to heat shock.

\section{Competitive fitness assays}

Relative fitness under benign conditions $\left(30^{\circ} \mathrm{C}\right)$ was measured as competitive fitness using a reference strain, YIR044C $\Delta$. All focal strains/test populations and the reference strain were grown to saturation in SDM+ (His, Met, Leu, Ura) at $30^{\circ} \mathrm{C}$ and resuspended using spectrophotometry to an optical density of 0.05 . Following the resuspension, the reference strain and individual focal strains/test populations were competed in SDM + (His, Met, Leu, Ura) for 48 hours. Proportions of focal strain/test population relative to the reference strain both before and after the competition were determined by plating on YPDA and YPDA+G418 (drug concentration of $200 \mu \mathrm{g} / \mathrm{ml}$ ), 
G418 obtained from Sigma-Aldrich). Relative fitness was calculated as per the protocol described in Wong, Rodrigue \& Kassen (2012) where the selection coefficient was determined using the equation described below. Fitness $w$ was calculated as $1+s$.

$$
s=\frac{\ln \frac{\text { focalinitial }}{\text { focalfinal }}-\ln \frac{\text { referenceinitial }}{\text { referencefinal }}}{\text { No. of generations }}
$$

\subsubsection{Experimental evolution:}

In this study, heat shock tolerance was evolved using the T1 isolate as the progenitor in 8 replicate lines across two sequential regimes, viz., Regime-A and RegimeB. Regime-A consisted of heat shocks applied every $\sim 8$ generations for $\sim 100$ generations (11 heat shocks over 32 days). At the end of Regime-A, the 8 replicate lines were transferred to Regime-B for another $\sim 100$ generations. Regime-B consisted of heat shocks applied every $\sim 40$ generations (2 heat shocks over 26 days). Because heat shocked cells take longer to resume growth, subsequent heat shocks in Regime-A were applied 72 hours after heat shocked cells were inoculated in fresh media. In Regime-B, cultures were diluted in fresh media five times ( $~ 8$ generations per dilution) between heat shocks. For the application of heat shocks, cells were first grown in SDM for 48 hours (Appendix A) following which a cell suspension was prepared by dilution to an $\mathrm{OD}_{600}$ of $0.05 .250 \mu \mathrm{L}$ of this cell suspension was pipetted into microcentrifuge tubes and heat shocked at $54^{\circ} \mathrm{C}$ for 75 minutes. $100 \mu \mathrm{L}$ of the heat shocked suspension was used to inoculate $5 \mathrm{ml}$ of fresh SDM. 


\subsubsection{Constraint estimation:}

Evolutionary constraints were measured phenotypically as the retention of heat shock tolerance under opposing selection (growth at $30^{\circ} \mathrm{C}$ ). Cells from the focal strains/test populations were grown in YPD in duplicate for 24 hours and diluted to an $\mathrm{OD}_{600}$ of 0.05 . This was repeated for 18 days ( 150 generations). Heat shock tolerance was measured at generation 0 , generation 100 and generation 150 following which statistical analyses were performed for the loss of heat shock tolerance. Fitness at $30^{\circ} \mathrm{C}$ was also measured at generation 0 and generation 150 following which statistical analyses were performed for gain in fitness under benign conditions.

\section{$\underline{\text { 2.2.5 }}$ Statistical analyses:}

Analysis of heat shock tolerance and competitive fitness for the ancestor T1 (6 trait measurements from independent replicate populations) and 8 replicate populations at the end of Regime-A (EoR-A lines) and at the end of Regime-B (EoR-B lines) were performed using one-way ANOVA tests. Post-hoc Tukey tests were performed to make pair-wise comparisons of heat shock tolerance and competitive fitness. Since replicate lines from EoR-A and EoR-B are independently evolving, we use a simple ANOVA model instead of a repeated measures model.

The two regimes (Regime-A and Regime-B) and three time points (generation 0 , generation 100 and generation 150) were used to perform statistical analyses of evolved constraints. A two-way ANOVA was used to model the effect of regimes and time points 
on heat shock tolerance, following which a post-hoc Tukey test was performed. A similar analysis was also performed using a Two-way ANOVA test to model the effect of regimes and time points on competitive fitness. A simpler ANOVA model is used here instead of a repeated measures model as individual replicate lines in this experiment evolve completely independently through time. Statistical packages used were R and Prism Graphpad.

\subsection{Results:}

\subsubsection{Heat shock tolerance evolves at the cost of fitness at $30^{\circ} \mathrm{C}$ in both regimes}

A one-way ANOVA (Table 2.1) showed that heat shock tolerance was significantly different across groups $(p=0.0017)$. A follow up post-hoc Tukey test (Table 2.2) revealed that evolved heat shock tolerance in lines from the end of Regime-A (EoR-A; $($ mean $=21.92 \%)$ and end of Regime-B (EoR-B; $($ mean $=19.77 \%)$ was significantly higher than that of the ancestor T1 (mean $=12.44 \%)$. However, the heat shock tolerance of lines from EoR-A and EoR-B did not significantly differ from each other $(p=0.576)$. A similar analysis using a one-way ANOVA (Table 2.3) to compare relative fitness under benign conditions revealed significant differences across groups. A follow up post-hoc Tukey test (Table 2.4) showed that relative fitness under benign conditions for lines from EoR-A $($ mean $=1.089)$ and EoR-B $($ mean $=1.038)$ was significantly lower relative to the ancestor T1 (mean =1.171). Here too, lines from EoR-A and EoR-B were not 
significantly different from each other in terms of relative fitness under benign conditions $(p=0.12)$.

\subsubsection{Analysis of the evolutionary constraintassay}

A two-factor ANOVA (Table 2.5) was used to model the effect of regime (that differed in the frequency of heat shocks) and time evolved under benign conditions in the final evolutionary constraint assay on heat shock tolerance. We found significant main effects of both regime $(p=0.004)$ and time evolved under benign conditions $(p=$ 0.0259). Interestingly, the interaction between regime and time was also significant ( $p=$ 0.004). Overall, lines from EoR-A lost heat shock tolerance more rapidly than lines from EoR-B (Fig. 2.2). A post-hoc Tukey test (Table 2.6) revealed a significant reduction in heat shock tolerance by generation 150 for lines from EoR-A ( $p=0.001)$. In contrast, heat shock tolerance did not significantly change by generation 150 for lines from EoR-B $(p=$ 0.988). Furthermore, we found an increasing effect of regime across time evolved under benign conditions as heat shock tolerance was greater for lines from EoR-B compared to lines from EoR-A only at generation $150(p=0.003)$ but not at generation $100(p=$ $0.299)$ and generation $0(p=0.945)$.

A two-factor ANOVA (Table 2.7) was used to model the effect of regimes that differ in the frequency of heat shocks and time evolved under benign conditions on fitness at $30{ }^{\circ} \mathrm{C}$. Here too, we found the effects of regime, time and the regime:time interaction to be significant $(p<0.001$ for both regime and time, $p=0.011$ for regime:time). Overall, lines from EoR-A increased fitness at $30^{\circ} \mathrm{C}$ more rapidly than lines 
from EoR-B (Fig. 2.2.B). A post-hoc Tukey test (Table 2.8) revealed a significant increase in fitness at $30^{\circ} \mathrm{C}$ by generation 150 for lines from EoR-A $(p<.001)$. In contrast, heat shock tolerance did not significantly change by generation 150 for lines from EoR-B ( $p=$ 0.342). We foundd an increasing effect of regime across time evolved under benign conditions as fitness at $30{ }^{\circ} \mathrm{C}$ was greater for lines from EoR-A compared to lines from EoR-B at generation $150(p<.001)$ but not at generation $0(p=0.649)$.

A

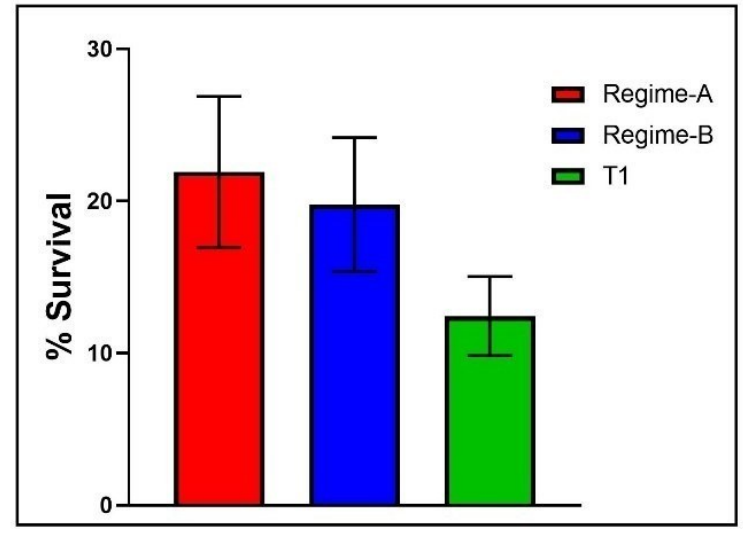

B

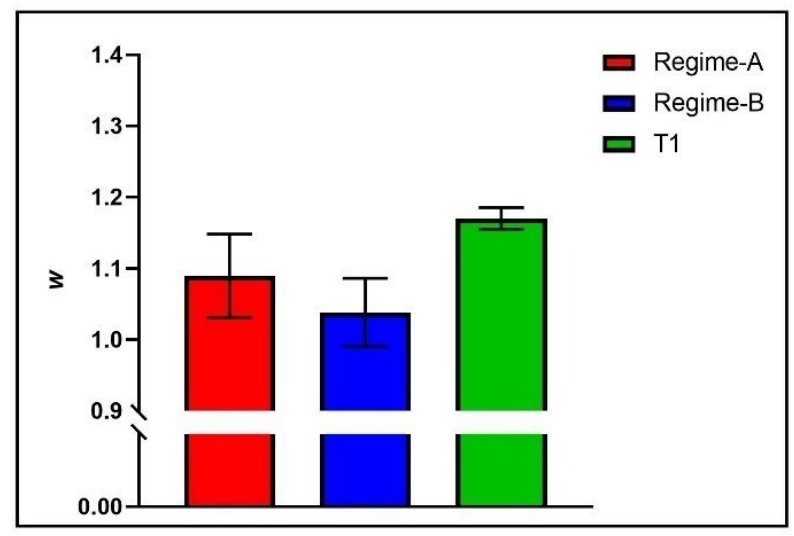

Figure 2.2: Trait measurements after evolution in Regime-A (100 generations) and Regime-B (100 generations) relative to the T1 ancestor. (A) Heat shock tolerance (measured as \% Survival) across lines. (B) Competitive fitness $(w)$ under benign conditions across lines. Error bars represent SD. 
A

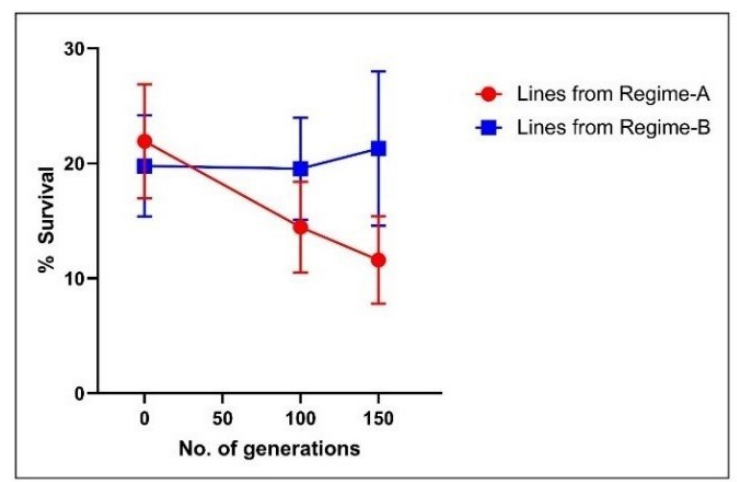

B

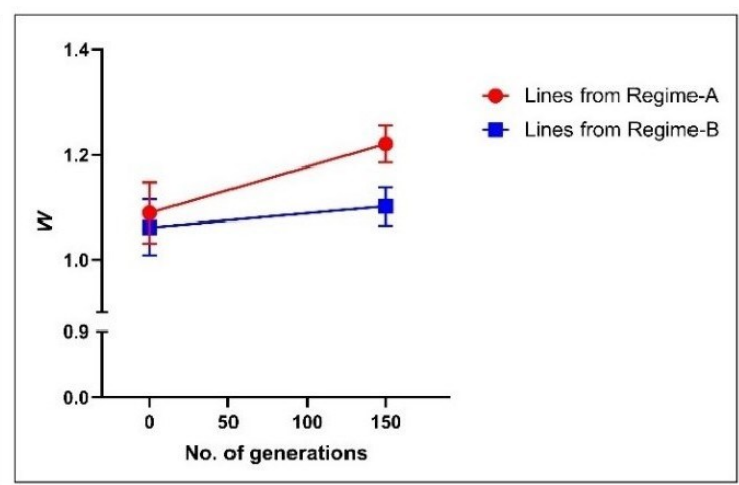

Figure 2.3: Evolved constraints measured as (A) loss of heat shock tolerance (measured as \% Survival) and (B) gain in competitive fitness at $30^{\circ} \mathrm{C}$ after selection in the evolutionary constraint assay under benign conditions for 150 generations in lines from Regime-A (red) and Regime-B (blue). Error bars represent SD.

Table 2. 1: Results of a one-way ANOVA for differences in heat shock tolerance across evolved lines from EoR-A \& EoR-B and the T1 ancestor.

\begin{tabular}{lcccc}
\hline \multicolumn{1}{c}{ Source } & $d f$ & $M S$ & $F$ & $P$ \\
\hline Between groups & 2 & 163.5 & 9.085 & 0.0017 \\
Within groups & 19 & 18.0 & & \\
Total & 21 & & & \\
\hline
\end{tabular}

Table 2. 2: Post-hoc Tukey test results for differences in heat shock tolerance across evolved lines and the T1 ancestor. Common letters indicate homogenous subsets. Letters that are not shared indicate significantly different mean values.

\begin{tabular}{|c|c|c|}
\hline Groups & $\begin{array}{l}\text { Letters showing homogenous } \\
\text { subsets }\end{array}$ & Mean HST (\% Survival) \\
\hline Regime-A & A & 21.92 \\
\hline Regime-B & A & 19.77 \\
\hline T1 & B & 12.44 \\
\hline
\end{tabular}

Table 2. 3: Results of a one-way ANOVA for differences in relative fitness under benign conditions across evolved lines from EoR-A \& EoR-B and the T1 ancestor. 


\begin{tabular}{lcccc}
\hline \multicolumn{1}{c}{ Source } & $d f$ & $M S$ & $F$ & $P$ \\
\hline Between groups & 2 & 0.02344 & 9.751 & 0.0015 \\
Within groups & 17 & 0.002404 & & \\
Total & 19 & & & \\
\hline
\end{tabular}

Table 2. 4: Post-hoc Tukey test results for differences in relative fitness under benign conditions across evolved lines and the T1 ancestor. Common letters indicate homogenous subsets. Letters that are not shared indicate significantly different mean values.

\begin{tabular}{|c|c|c|}
\hline Groups & $\begin{array}{l}\text { Letters showing homogenous } \\
\text { subsets }\end{array}$ & Mean fitness \\
\hline Regime-A & A & 1.089 \\
\hline Regime-B & A & 1.038 \\
\hline T1 & B & 1.17 \\
\hline
\end{tabular}

Table 2. 5: Results of a two-factor ANOVA for differences in heat shock tolerance across evolution regimes and time points. Lines from EoR-A and EoR-B and time points at generation 0, 100 and 150 of the evolutionary constraint assay were compared.

\begin{tabular}{lcccc}
\hline \multicolumn{1}{c}{ Source } & $d f$ & SS & $F$ & $P$ \\
\hline Regime & 1 & 212.8 & 9.195 & 0.0042 \\
Time & 2 & 184.7 & 3.991 & 0.0259 \\
Regime: Time & 2 & 285.7 & 6.174 & 0.0045 \\
Residuals & 42 & 971.9 & & \\
\hline
\end{tabular}


Table 2. 6: Results of a post-hoc Tukey test for differences in heat shock tolerance at different timepoints for lines from EoR-A and EoR-B. Common letters indicate homogenous subsets. Letters that are not shared indicate significantly different mean values.

\begin{tabular}{|c|c|c|c|c|}
\hline Level (Strain : Time) & \multicolumn{3}{|c|}{ Letters showing homogenous subsets } & Mean HST \\
\hline Regime-A : Gen0 & \multicolumn{3}{|l|}{ A } & 21.92 \\
\hline Regime-B : Gen0 & $A$ & \multicolumn{2}{|l|}{ B } & 19.77 \\
\hline Regime-B : Gen100 & A & \multicolumn{2}{|l|}{ B } & 19.53 \\
\hline Regime-B : Gen100 & $A$ & \multicolumn{2}{|l|}{ B } & 21.28 \\
\hline Regime-A : Gen 100 & & \multirow[t]{2}{*}{ B } & C & 14.45 \\
\hline Regime-A : Gen150 & & & C & 11.58 \\
\hline Source & $d f$ & SS & $F$ & $P$ \\
\hline Regime & 1 & 0.043 & 19.207 & $<.001$ \\
\hline Time & 1 & 0.059 & 26.331 & $<.001$ \\
\hline Regime: Time & 1 & 0.017 & 7.423 & 0.011 \\
\hline Residuals & 28 & 0.002 & & \\
\hline
\end{tabular}

Table 2. 8: Results of a post-hoc Tukey test for differences in fitness at $30^{\circ} \mathrm{C}$ at different timepoints for lines from EoR-A and EoR-B. Common letters indicate homogenous subsets. Letters that are not shared indicate significantly different mean values.

\begin{tabular}{lcc}
\hline Level (Strain : Time) & $\begin{array}{c}\text { Letters showing } \\
\text { homogenous subsets }\end{array}$ & Mean fitness \\
\hline Regime-A : Gen0 & A & 1.090 \\
Regime-B : Gen0 & A & 1.062 \\
Regime-B : Gen150 & A & 1.102 \\
Regime-A : Gen150 & & B \\
\hline
\end{tabular}




\subsection{Discussion:}

Although bet hedging is known to evolve in unpredictably fluctuating environments, how it is expected to evolve when it involves selection for apparent suboptimality over shorter time scales remains an unanswered question. Under conditions where bet hedging is expected to evolve, long-term evolutionary success is maximized by the ability to retain traits that increase survival in rare but detrimental environments. This is only possible if there is a lack of response to selection under benign conditions. In this study, we test the hypothesis that such adaptive constraints on short-term evolution evolve. We take an experimental evolution approach, using fluctuating selection comprised of heat shocks and benign environments imposed across two sequential regimes (Regime-A and Regime-B) in which the frequency of heat shocks decreases. Following evolution in both regimes, we expect the evolution of increased heat shock tolerance that comes at a cost of fitness under benign conditions.

To test the central hypothesis that bet hedging evolves as a constraint on adaptive tracking, we empirically measured evolved constraints in lines from the end of Regime-A (EoR-A) and the end of Regime-B (EoR-B) by allowing for further evolution under constant benign conditions and assaying periodically for the loss of heat shock tolerance and increase in fitness at $30^{\circ} \mathrm{C}$. If bet hedging does evolve as a constraint on adaptive tracking, we expect heat shock tolerance to be retained in lines from EoR-B (where heat shocks were rare) longer than for lines from EoR-A (where heat shocks were frequent). This is what we find as lines from EoR-A lose heat shock tolerance over 150 generations whereas lines from EoR-B do not. We also find that the loss of heat 
shock tolerance in lines from EoR-A is associated with an increase in fitness at $30^{\circ} \mathrm{C}$. In contrast, lines from EoR-B do not significantly improve fitness at $30^{\circ} \mathrm{C}$ after evolving for 150 generations under benign conditions. This indicates that evolution in Regime-B, where heat shocks were rare, selected for a constraint on adaptive tracking that prevented the otherwise expected loss of heat shock tolerance and concomitant increase in fitness under benign conditions. Furthermore, after evolving for 150 generations under benign conditions, lines from EoR-B, while suboptimal under benign conditions, have a significantly greater heat shock tolerance relative to lines from EoR-A.

Evolved lines from EoR-A and Eor-B had a higher heat shock tolerance and lower fitness under benign conditions relative to the ancestor, suggesting that heat shock tolerance came at the cost of fitness under benign conditions. Due to the greater number of benign environments in Regime-B, lines from EoR-B were expected to have lower heat shock tolerance and higher fitness under benign conditions relative to lines from EoR-A. However, lines from EoR-A and EoR-B did not significantly differ in heat shock tolerance or in fitness under benign conditions possibly because the lines in Regime-B were only evolved for a relatively short period of time (100 generations). Relative fitness under benign conditions was measured using competition assays with a reference strain. It is important to note that due to auxotrophies in the reference strain, assays were performed in medium enriched with the necessary amino acids. Therefore, if genotype-environment interactions exist between evolved yeast strains and the defined, amino acid enriched medium, estimates of relative fitness may not accurately represent fitness under experimental conditions imposed during evolution. To account 
for background effects of laboratory conditions across sequential regimes, a control line was evolved under benign conditions for the duration of both Regime-A and Regime-B. The two control lines were assayed for heat shock tolerance and growth under benign conditions and were found to not significantly differ from each other, indicating that background laboratory conditions did not influence evolved heat shock tolerance or growth under benign conditions.

Maximizing the GMF, or long-term evolutionary success, is strongly influenced by fitness in rare but extreme environments. Because constraints on adaptive tracking result in increased survival under such detrimental conditions, they can potentially be adaptive in the long-term. Constraints on tracking cannot evolve instantaneously and, like all selectable traits, genetic variation in constraint is a prerequisite for the evolution of a constraint. While substantial phenotypic variation in constraint was observed at the end of both Regime-A and Regime- $B$, genetic variation for constraint could not be quantified using this study design. Future study aiming to estimate genetic variation in constraint would require replication of individual lines in assays for evolved constraints.

Some genetic mechanisms behind bet-hedging traits have been described (Evans, Dionne, Miller, \& Bernatchez, 2012; Maxwell \& Magwene, 2017; Simons \& Johnston, 2006; Verstraeten et al., 2015). In yeast, the trait that controls heat shock tolerance has been shown to be a diversification bet-hedging strategy (S. F. Levy, Ziv, \& Siegal, 2012). Clonal yeast populations show a distribution of growth rates when cultured in benign environments (Avery, 2006; Raser \& O’Shea, 2004). Slow growth rates are correlated with intracellular levels of Tsl1, a regulator of trehalose synthesis, 
and are a reliable predictor of heat shock tolerance (Levy, Ziv \& Siegal, 2012). However, despite an understanding of genetic mechanisms underlying bet-hedging traits, the genetic basis of evolutionary constraints that influence the persistence of bet-hedging traits remains poorly understood. The present study warrants genetic studies of evolved constraints to better understand how bet-hedging traits persist in the short-term. Longterm selection acting on constrained traits can potentially lead to such traits being phylogenetically embedded and shared between multiple species. In that case, evolutionarily conserved traits could be considered a product of long-term selection for constrained traits. An example of a conserved trait in yeast are orthologous ribosomal protein promoters that show conserved expression even when genomically integrated into other distinct yeast species. This conserved expression was found to be mediated by compensatory changes where the effects of mutations in one site of the core promoter are nullified by mutations in another site (Zeevi et al., 2014).

Our study provides qualitative evidence that the evolution of bet hedging occurs through selection for a constraint on adaptive tracking. However, quantitative inferences of the degree of constrained evolution that is adaptive given the frequency of heat shocks is beyond the scope of this study, and would require detailed assessment of the optimal value of the constrained trait that maximizes geometric-mean fitness across fluctuating environments. This study shows that the retention of heat shock tolerance only occurred in Regime-B, where heat shocks were rare, demonstrating that selection can act on variation in heat shock tolerance retention which is necessary for bet 
hedging. Although constraints have traditionally been viewed as maladaptive, we show that they may be adaptive and could potentially be selected for over the long term. 


\section{Appendices}

\section{Appendix A: Heat shock tolerance across phases of yeast growth}

The use of heat shock in an evolution regime requires an understanding of the appropriate timing of heat shocks to ensure consistency of heat shock tolerance measurements and to maximize the difference in heat shock tolerance between thermotolerant variants and the wild type ancestor. Yeast growth occurs across three distinct growth phases, viz. lag, exponential growth and saturation. Cells in the saturation or $\mathrm{G}_{0}$ phase have been found to be significantly more thermotolerant due to higher levels of trehalose accumulation than for rapidly dividing cells (Paris \& Pringle, 1983). To understand how tolerance to heat shocks varies across different phases of growth, we administered heat shocks at 4 hour intervals over a period of 48 hours for the ancestor S288C and the evolved thermotolerant T1 strain (Fig. A1). Heat shock tolerance at each time point was measured in duplicate as the percentage of surviving cells relative to the total number of cells. We found that although heat shock tolerance varied over 48 hours, it stabilized around 36 to 48 hours. Moreover, the difference in heat shock tolerance between the thermotolerant strain (T1) and the wild type (S288C) was greatest around 36-48 hours (late saturation phase) compared to the lag phase (0 to 8 hours) and the exponential growth phase ( 8 to 16 hours). Using this information, heat shock tolerance assays were designed such that assays were performed after 48 
hours of growth to ensure consistency in heat shock tolerance measurements. Evolution regimes using heat shocks were designed such that heat shocks occurred at the late saturation phase of growth in order to maximize the difference in survival between thermotolerant variants and theancestor.

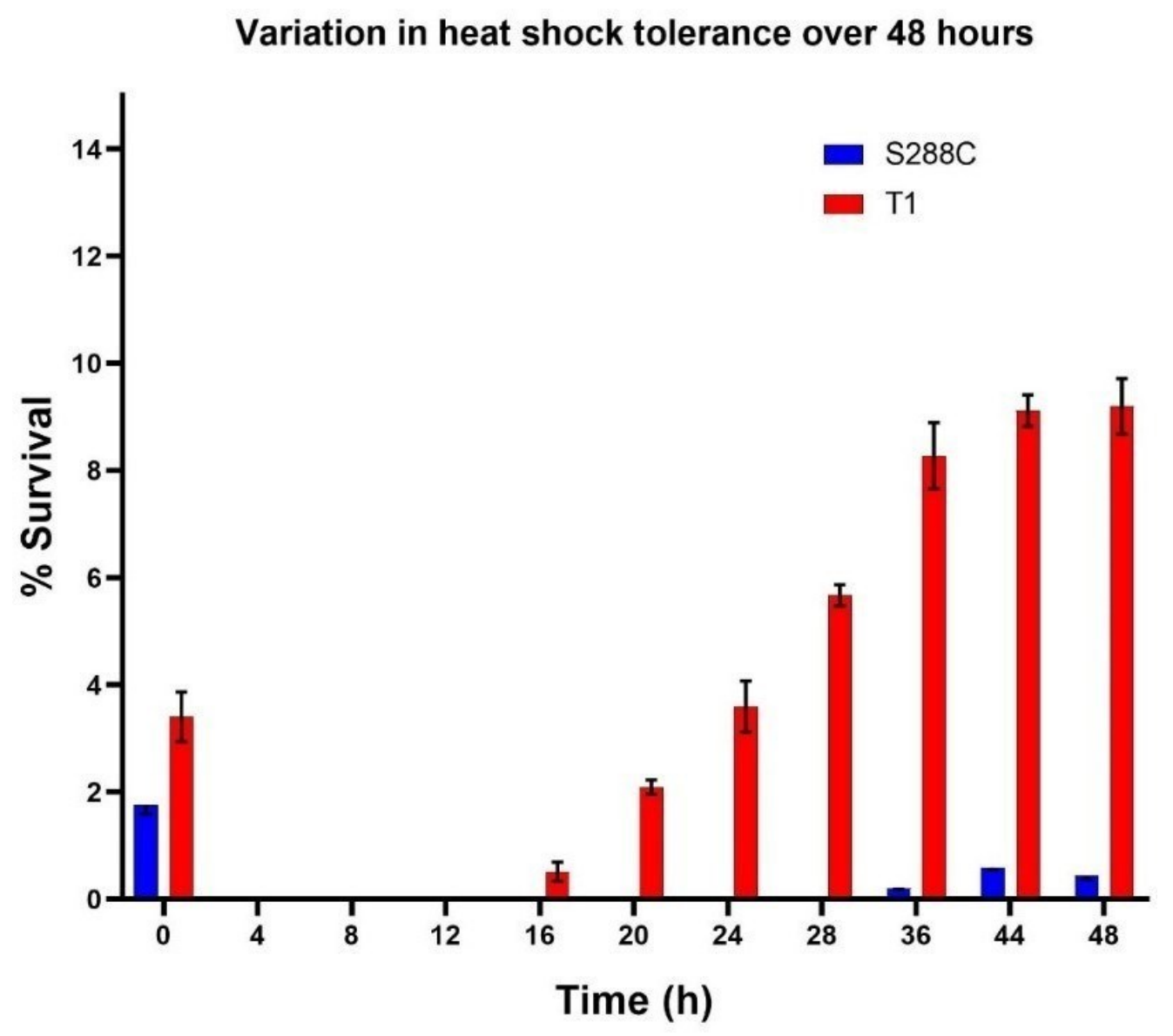

Figure A.1: Heat shock tolerance (expressed as \% Survival) measured at 4 hour intervals over 48 hours for S288C and T1 strains. Error bars represent SD. 


\section{Appendix B: Estimation of evolutionary constraint}

Testing the hypothesis that bet hedging evolves as a constraint on adaptive tracking requires an appropriate method to measure evolutionary constraints. Because evolutionary constraints offer resistance along the course of adaptation, they can be measured empirically as the persistence of a trait despite selection acting against the trait (Arnold, 1992). If heat shock tolerance comes at a fitness cost under benign conditions, continued evolution under benign conditions should result in the loss of heat shock tolerance. However, if adaptation to benign conditions is constrained, the retention of heat shock tolerance is expected. As a preliminary test to determine how best to measure constraints associated with evolved heat shock tolerance, the two isolates (Iso-1, Iso-2) from the heat shock regime and their ancestor, the T1 strain (Chapter 1, Section 1.1.3), were evolved for 100 generations under constant benign conditions $\left(30^{\circ} \mathrm{C}\right)$ in 3 replicate lines each. Because the T1 strain was derived from a population that was evolved at $40^{\circ} \mathrm{C}$ whereas the two isolates were evolved under repeated heat shocks, predictions regarding differences evolved constraints between the T1 strain and the isolates could notbemade.

Heat shock tolerance and fitness under benign conditions was measured at generation 0 and generation 100. Fitness under benign conditions was measured as competitive fitness using a reference strain. Our results demonstrate that evolution under benign conditions resulted in a significant reduction of heat shock tolerance in 
Iso- 1 and Iso- 2 after only 100 generations. The T1 strain, in contrast, did not show a significant reduction in heat-shock tolerance. Furthermore, we also find that fitness under benign conditions (measured as competitive fitness) increased for Iso-1 and Iso-2 but not for T1 after further evolution under benign conditions. Taken together, these results suggest that evolution under benign conditions is more constrained for T1 compared to Iso-1 and Iso-2.

A

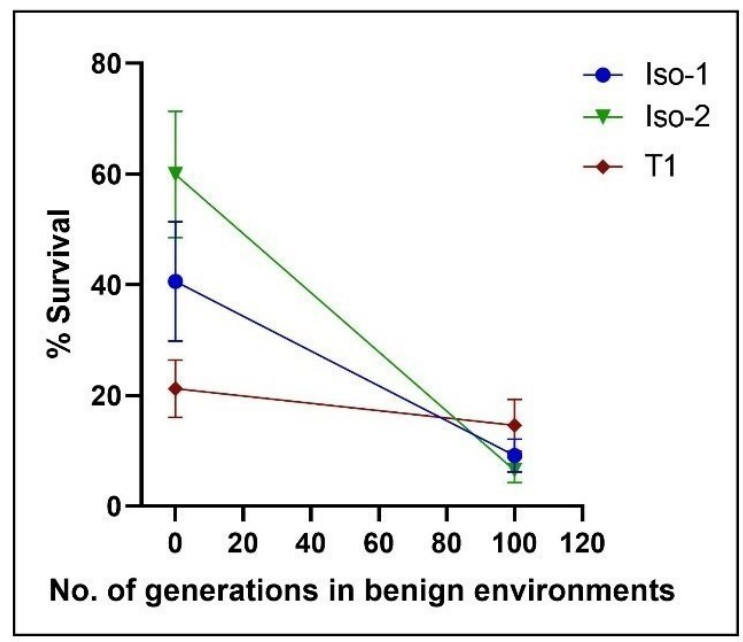

B

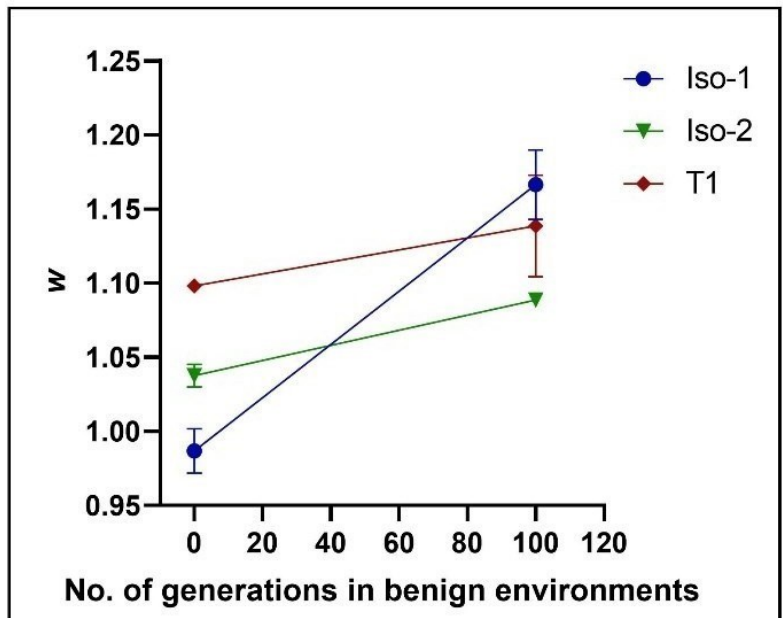

Figure B.1: : Evolved constraints empirically measured as (A) loss of heat shock tolerance (measured as \% Survival) and (B) gain in fitness under benign conditions after selection under benign conditions for 100 generations in the isolates Iso-1 (blue), Iso-2 (green) and the ancestor T1 (red). Error bars represent SD. 


\section{Appendix C: Estimation of doubling time and number of generations per transfer}

To estimate the number of generations per transfer during experimental evolution, we calculated the doubling time from growth measurements. Two mid-log values were used to calculate doubling time. Here, we used $\mathrm{OD}_{600}$ measurements at 15.5 hours (0.402) and 21 hours (1.609). Doubling time was calculates using the equation:

$$
\text { DoublingTime }=\frac{\text { duration } * \log (2)}{\log (\text { FinalConcentration })-\log (\text { InitalConcentration })} .
$$

Using this, doubling time was estimated to be 2.74 hours. We the use the doubling time to estimate the number of doublings, given the starting cell concentration, for a cell culture to reach early saturation. Since yeast cultures reach saturation $\sim 22.5$ hours, the number of generations $(\mathrm{g})$ in each transfer during experimental evolution was calculated as $g=T / T_{d}$.

$T$ is the time taken to reach early saturation and $T_{d}$ is the doubling time. Using this, $g$ was calculated to be 8.213 . 


\section{Literature cited}

Agrawal, A. A., Conner, J. K., \& Rasmann, S. (2010). Tradeoffs and negative correlations in evolutionary ecology. In Evolution since Darwin: the first 150 years, 243-268.

Anderson, J. B., Sirjusingh, C., Parsons, A. B., Boone, C., Wickens, C., Cowen, L. E., \& Kohn, L. M. (2003). Mode of selection and experimental evolution of antifungal drug resistance in Saccharomyces cerevisiae. Genetics, 163(4): 1287-1298.

Arnold, S. J. (1992). Constraints on phenotypic evolution. The American Naturalist, 140: S85-S107.

Avery, S. V. (2006). Microbial cell individuality and the underlying sources of heterogeneity, Nature Reviews Microbiology, 4: 577-587.

Balmford, A. (1996). Extinction filters and current resilience: The significance of past selection pressures for conservation biology. Trends in Ecology and Evolution, 11: 193-196.

Beaumont, H. J. E., Gallie, J., Kost, C., Ferguson, G. C., \& Rainey, P. B. (2009). Experimental evolution of bet hedging. Nature, 462(7269): 90-93.

Ben-Ami, R., Garcia-Effron, G., Lewis, R. E., Gamarra, S., Leventakos, K., Perlin, D. S., \& Kontoyiannis, D. P. (2011). Fitness and virulence costs of Candida albicans FKS1 hot spot mutations associated with echinocandin resistance. Journal of Infectious Diseases, 204(4): 626-635. 
Bennett, A. F., \& Lenski, R. E. (2007). An experimental test of evolutionary trade-offs during temperature adaptation. Proceedings of the National Academy of Sciences, 104 (Supp 1): 8649-8654.

https://doi.org/10.17226/11790

Berry, D. B., \& Gasch, A. P. (2008). Stress-activated genomic expression changes serve a preparative role for impending stress in yeast. Molecular Biology of the Cell, 19(11): 4580-4587.

https://doi.org/10.1091/mbc.E07-07-0680

Blows, M. W., \& Hoffmann, A. A. (2005). A reassessment of genetic limits to evolutionary change. Ecology, 86(6): 1371-1384. https://doi.org/10.1890/04-1209

Botero, C. A., Weissing, F. J., Wright, J., \& Rubenstein, D. R. (2015). Evolutionary tipping points in the capacity to adapt to environmental change. Proceedings of the National Academy of Sciences, 112(1): 184-189.

https://doi.org/10.1073/pnas.1408589111

Bradford, M. J., \& Roff, D. A. (1993). Bet hedging and the diapause strategies of the cricket Allonemobius fasciatus. Ecology, 74(4): 1129-1135.

https://doi.org/10.2307/1940482

Caspeta, L., Chen, Y., Ghiaci, P., Feizi, A., Baskov, S., Hallström, B. M., Petranovic, D. \& Nielsen, J. (2014). Altered sterol composition renders yeast thermotolerant. Science, 356 (6205): 75-78. https://doi.org/10.1126/science.1258137 
Caspeta, L., Chen, Y., \& Nielsen, J. (2016). Thermotolerant yeasts selected by adaptive evolution express heat stress response at $30^{\circ} \mathrm{C}$. Scientific Reports, 6: 27003. http://dx.doi.org/10.1038/srep27003

Caspeta, L., \& Nielsen, J. (2015). Thermotolerant yeast strains adapted by laboratory evolution show trade-off at ancestral temperatures and preadaptation to other stresses. MBio, 6: e00431-15.

http://mbio.asm.org/content/6/4/e00431-15.abstract

Castrillo, J. I., Zeef, L. A., Hoyle, D. C., Zhang, N., Hayes, A., Gardner, D. C. J., Cornell, M. J., Petty, J., Hakes, L., Wardleworth, L., Rash, B., Dunn, W.B., Broadhurst, D., O'Donoghue, K., Hester, S. S., Dunkley, T. P., Hart, S. R., Swainston, N., Li, P., Paton, N. W., Lilley, K. S., Kell, D. B. \& Oliver, S. G. (2007). Growth control of the eukaryote cell: A systems biology study in yeast. Journal of Biology, 6(2):4. https://doi.org/10.1186/jbiol54

Childs, D. Z., Metcalf, C. J. E., \& Rees, M. (2010). Evolutionary bet-hedging in the real world: Empirical evidence and challenges revealed by plants. Proceedings of the Royal Society B: Biological Sciences, 277(1697): 3055-3064. https://doi.org/10.1098/rspb.2010.0707

Clark, J. S., Dietze, M., Chakraborty, S., Agarwal, P. K., Ibanez, I., LaDeau, S., \& Wolosin, M. (2007). Resolving the biodiversity paradox. Ecology Letters, 10(8): 647-659 https://doi.org/10.1111/j.1461-0248.2007.01041.x

Cohen, D. (1966). Optimizing reproduction in a randomly varying environment. Journal 
of Theoretical Biology, 12(1): 119-129.

https://doi.org/10.1016/0022-5193(66)90188-3

Cowen, L. E., Kohn, L. M., \& Anderson, J. B. (2001). Divergence in fitness and evolution of drug resistance in experimental populations of Candida albicans. J Bacteriol, 183(10): 2971-2978.

https://doi.org/10.1128/JB.183.10.2971

Cowen, L. E., Sanglard, D., Howard, S. J., Rogers, P. D., \& Perlin, D. S. (2014). Mechanisms of antifungal drug resistance. Cold Spring Harbor Perspectives in Medicine, 5(7): a019752.

https://doi.org/10.1101/cshperspect.a019752

Craig, E. A., Gambill, B. D., \& Nelson, R. J. (1993). Heat shock proteins: Molecular chaperones of protein biogenesis. Microbiological Reviews, 57(2): 402-414.

De Virgilio, C., Hottiger, T., Dominguez, J., Boller, T., \& Wiemken, A. (1994). The role of trehalose synthesis for the acquisition of thermotolerance in yeast: I. Genetic evidence that trehalose is a thermoprotectant. European Journal of Biochemistry, 219(1-2): 179-186.

https://doi.org/10.1111/j.1432-1033.1994.tb19928.x

Dempster, E. R. (1955). Maintenance of genetic heterogeneity. Cold Spring Harbor Symposia on Quantitative Biology, 20: 25-31. https://doi.org/10.1101/SQB.1955.020.01.005 
Deutscher, D., Meilijson, I., Kupiec, M., \& Ruppin, E. (2006). Multiple knockout analysis of genetic robustness in the yeast metabolic network. Nature Genetics, 38: 993998. https://doi.org/10.1038/ng1856

Dudley, A. M., Janse, D. M., Tanay, A., Shamir, R., \& Church, G. M. (2005). A global view of pleiotropy and phenotypically derived gene function in yeast. Molecular Systems Biology, 1(1): E1-E11. https://doi.org/10.1038/msb4100004

Dunham, M. J., Badrane, H., Ferea, T., Adams, J., Brown, P. O., Rosenzweig, F., \& Botstein, D. (2002). Characteristic genome rearrangements in experimental evolution of Saccharomyces cerevisiae. Proceedings of the National Academy of Sciences of the United States of America, 99(25): 16144-16149. https://doi.org/10.1073/pnas.242624799

Edgardo, A., Carolina, P., Manuel, R., Juanita, F., \& Baeza, J. (2008). Selection of thermotolerant yeast strains Saccharomyces cerevisiae for bioethanol production. Enzyme and Microbial Technology, 43(2): 120-123. https://doi.org/10.1016/J.ENZMICTEC.2008.02.007

Elena, S. F., \& Lenski, R. E. (2003). Evolution experiments with microorganisms: The dynamics and genetic bases of adaptation. Nature Reviews Genetics, 4: 457-469. https://doi.org/10.1038/nrg1088

Eleutherio, E. C. A., Araujo, P. S., \& Panek, A. D. (1993). Protective role of trehalose during heat stress in Saccharomyces cerevisiae. Cryobiology, 30(6): 591-596. https://doi.org/10.1006/cryo.1993.1061 
Elliott, B., Haltiwanger, R. S., \& Futcher, B. (1996). Synergy between trehalose and Hsp104 for thermotolerance in Saccharomyces cerevisiae. Genetics, 144(3): 923933.

Engel, S. R., Balakrishnan, R., Binkley, G., Christie, K. R., Costanzo, M. C., Dwight, S. S., Fisk, D. G., Hirschman, J. E., Hitz, B. C., Hong, E. L., Krieger, C. J., Licstone, M., S., Miyasato, S. R., Nash, R., Oughtred, R., Park, J., Skrzypek, M. S., Weng, S., Wong, E. D., Dolinski, K., Botstein, D. \& Cherry, J. M. (2009). Saccharomyces Genome Database provides mutant phenotype data. Nucleic Acids Research, 38(SUPPL.1): 433-436. https://doi.org/10.1093/nar/gkp917

Evans, M. L., Dionne, M., Miller, K. M., \& Bernatchez, L. (2012). Mate choice for major histocompatibility complex genetic divergence as a bet-hedging strategy in the atlantic salmon (Salmo salar). Proceedings of the Royal Society B: Biological Sciences, 279: 379-386. https://doi.org/10.1098/rspb.2011.0909

Ferea, T. L., Botstein, D., Brown, P. O., \& Rosenzweig, R. F. (1999). Systematic changes in gene expression patterns following adaptive evolution in yeast. Proceedings of the National Academy of Sciences of the United States of America, 96(17): 9721-9726 https://doi.org/10.1073/pnas.96.17.9721

Fernandez-Ricaud, L., Warringer, J., Ericson, E., Glaab, K., Davidsson, P., Nilsson, F., Kemp, G. J. L., Nerman, O. \& Blomberg, A. (2007). PROPHECY -- a yeast phenome database, update 2006. Nucleic Acids Research, 35(Database issue): D463-467. https://doi.org/10.1093/nar/gkl1029 
Frankenhuis, W. E., Panchanathan, K., \& Belsky, J. (2016). A mathematical model of the evolution of individual differences in developmental plasticity arising through parental bet-hedging. Developmental Science, 19(2): 251-274.

https://doi.org/10.1111/desc.12309

Furness, A. I., Lee, K., \& Reznick, D. N. (2015). Adaptation in a variable environment: Phenotypic plasticity and bet-hedging during egg diapause and hatching in an annual killifish. Evolution, 69(6): 1461-1475. https://doi.org/10.1111/evo.12669

García-Roger, E. M., Serra, M., \& Carmona, M. J. (2014). Bet-hedging in diapausing egg hatching of temporary rotifer populations - A review of models and new insights. International Review of Hydrobiology, 99(1-2): 96-106. https://doi.org/10.1002/iroh.201301708

Garland, T. (2014). Trade-offs. Current Biology, 24(2): R60-R61. https://doi.org/10.1016/J.CUB.2013.11.036

Gillespie, J. H. (1977). Natural selection for variances in offspring numbers: A new evolutionary principle. The American Naturalist, 111: 1010-1014. https://doi.org/10.1086/283230

Graham, J. K., Smith, M. L., \& Simons, A. M. (2014). Experimental evolution of bet hedging under manipulated environmental uncertainty in Neurospora crassa. Proceedings of the Royal Society B-Biological Sciences, 281(1787): pii: 20140706.

Gremer, J. R., Kimball, S., \& Venable, D. L. (2016). Within-and among-year germination 
in Sonoran Desert winter annuals: bet hedging and predictive germination in a variable environment. Ecology Letters, 19(10): 1209-1218.

https://doi.org/10.1111/ele.12655

Hill, J. A., O'Meara, T. R., \& Cowen, L. E. (2015). Fitness trade-offs associated with the evolution of resistance to antifungal drug combinations. Cell Reports, 10;10(5): 809819.

https://doi.org/10.1016/j.celrep.2015.01.009

Huang, M., \& Kao, K. C. (2012). Population dynamics and the evolution of antifungal drug resistance in Candida albicans. FEMS Microbiology Letters, 333(2): 85-93. https://doi.org/10.1111/j.1574-6968.2012.02587.x

Huey, R. B., \& Kingsolver, J. G. (1989). Evolution of thermal sensitivity of ectotherm performance. Trends in Ecology and Evolution, 4(5): 131-135. https://doi.org/10.1016/0169-5347(89)90211-5

Kanafani, Z. A., \& Perfect, J. R. (2008). Resistance to antifungal agents: Mechanisms and clinical impact. Clinical Infectious Diseases, 46(1): 120-128. https://doi.org/10.1086/524071

Karve, S. M., Bhave, D., \& Dey, S. (2018). Extent of adaptation is not limited by unpredictability of the environment in laboratory populations of Escherichia coli. Journal of Evolutionary Biology, 31(9): 1420-1426.

https://doi.org/10.1111/jeb.13338 
Kassen, R. (2009). Toward a general theory of adaptive radiation: Insights from microbial experimental evolution. Annals of the New York Academy of Sciences, 1168: 3-22. https://doi.org/10.1111/j.1749-6632.2009.04574.x

Kawecki, T. J., Lenski, R. E., Ebert, D., Hollis, B., Olivieri, I., \& Whitlock, M. C. (2012). Experimental evolution. Trends in Ecology and Evolution, 27(10): 547-560. https://doi.org/10.1016/j.tree.2012.06.001

Knies, J. L., Izem, R., Supler, K. L., Kingsolver, J. G., \& Burch, C. L. (2006). The genetic basis of thermal reaction norm evolution in lab and natural phage populations. PLoS Biology, 4(7): e201. https://doi.org/10.1371/journal.pbio.0040201

Kodedová, M., \& Sychrová, H. (2015). Changes in the sterol composition of the plasma membrane affect membrane potential, salt tolerance and the activity of multidrug resistance pumps in Saccharomyces cerevisiae. PLoS ONE, 10(9): e0139306. https://doi.org/10.1371/journal.pone.0139306

Kolecka, A., Krauke, Y., Bujdakova, H., \& Sychrova, H. (2009). Subinhibitory concentrations of fluconazole increase the intracellular sodium content in both fluconazole-resistant and -sensitive Candida albicans strains. Canadian Journal of Microbiology, 55(5): 605-610. https://doi.org/10.1139/W09-009

Kültz, D. (2005). Molecular and evolutionary basis of the cellular stress response. Annual Review of Physiology, 67:225-257. 
https://doi.org/10.1146/annurev.physiol.67.040403.103635

Lee, M. S. Y., \& Doughty, P. (2003). The geometric meaning of macroevolution. Trends in Ecology and Evolution, 18(6): 263-266. https://doi.org/10.1016/S01695347(03)00103-4

Leu, J. Y., \& Murray, A. W. (2006). Experimental evolution of mating discrimination in budding yeast. Current Biology, 16(3): 280-286. https://doi.org/10.1016/j.cub.2005.12.028

Levin, B. R., Perrot, V., \& Walker, N. (2000). Compensatory mutations, antibiotic resistance and the population genetics of adaptive evolution in bacteria. Genetics, 154(3): 985-957.

Levy, S. F., Ziv, N., \& Siegal, M. L. (2012). Bet hedging in yeast by heterogeneous, agecorrelated expression of a stress protectant. PLoS Biol, 10(5): e1001325. https://doi.org/10.1371/journal.pbio.1001325

Levy, S., Ihmels, J., Carmi, M., Weinberger, A., Friedlander, G., \& Barkai, N. (2007). Strategy of transcription regulation in the budding yeast. PLOS ONE, 2(2): e250. https://doi.org/10.1371/journal.pone.0000250

Lewontin, R. C., \& Cohen, D. (1969). On population growth in a randomly varying environment. Proceedings of the National Academy of Sciences of the United States of America, 64(2): 1056-1060. https://doi.org/10.1073/pnas.62.4.1056

Louis, E. J. (2016). Historical evolution of laboratory strains of Saccharomyces cerevisiae. 


\section{Cold Spring Harbor Protocols, 7.}

https://doi.org/10.1101/pdb.top077750

Lupetti, A., Danesi, R., Campa, M., Tacca, M. Del, \& Kelly, S. (2002). Molecular basis of resistance to azole antifungals. Trends in Molecular Medicine, 8(2): 76-81. https://doi.org/10.1016/S1471-4914(02)02280-3

Lycus, P., Soriano-Laguna, M. J., Kjos, M., Richardson, D. J., Gates, A. J., Milligan, D. A., ... Bakken, L. R. (2018). A bet-hedging strategy for denitrifying bacteria curtails their release of $\mathrm{N}_{2}$ O. Proceedings of the National Academy of Sciences, 115(46): 1182011825. https://doi.org/10.1073/pnas.1805000115

Maisnier-Patin, S., \& Andersson, D. I. (2004). Adaptation to the deleterious effects of antimicrobial drug resistance mutations by compensatory evolution. Research in Microbiology, 155(5): 360-369. https://doi.org/10.1016/j.resmic.2004.01.019

Maxwell, C. S., \& Magwene, P. M. (2017). When sensing is gambling: An experimental system reveals how plasticity can generate tunable bet-hedging strategies.

Evolution, 71(4): 859-871. https://doi.org/10.1111/evo.13199

McGinley, M. A., \& Charnov, E. L. (1988). Multiple resources and the optimal balance between size and number of offspring. Evolutionary Ecology, 2: (77-84). https://doi.org/10.1007/BF02071590

Mortimer, R. K., \& Johnston, J. R. (1986). Genealogy of principal strains of the yeast 
genetic stock center. Genetics, 113(1): 35-43.

Orr, H. A. (2009). Fitness and its role in evolutionary genetics. Nature Reviews Genetics, 10(8): 531-539.

https://doi.org/10.1038/nrg2603

Paris, S., \& Pringle, J. R. (1983). Saccharomyces cerevisiae: Heat and gluculase sensitivities of starved cells. Annales de Microbiologie, 134B(3): 379-385.

Perlin, D. S., Rautemaa-Richardson, R., \& Alastruey-Izquierdo, A. (2017). The global problem of antifungal resistance: prevalence, mechanisms, and management. The Lancet Infectious Diseases, 17(12): e383-e392.

https://doi.org/10.1016/S1473-3099(17)30316-X

Philippi, T., \& Seger, J. (1989). Hedging one's evolutionary bets, revisited. Trends in Ecology and Evolution, 4(2): 41-44. https://doi.org/10.1016/0169-5347(89)90138-9

Piper, P. W. (1993). Molecular events associated with acquisition of heat tolerance by the yeast Saccharomyces cerevisiae. FEMS Microbiology Reviews, 11(4): 339-355. https://doi.org/10.1111/j.1574-6976.1993.tb00005.x

Rainey, P. B., Beaumont, H. J. E., Ferguson, G. C., Gallie, J., Kost, C., Libby, E., \& Zhang, X. X. (2011). The evolutionary emergence of stochastic phenotype switching in bacteria. Microbial Cell Factories, Suppl 1: S14. https://doi.org/10.1186/1475-2859-10-S1-S14

Rajon, E., Desouhant, E., Chevalier, M., Débias, F., \& Menu, F. (2014). The evolution of 
bet hedging in response to local ecological conditions. The American Naturalist, 184(1): E1-E15. https://doi.org/10.1086/676506

Raser, J. M., \& O'Shea, E. K. (2004). Control of stochasticity in eukaryotic gene expression. Science, 304(5678): 1811-1814. https://doi.org/10.1126/science.1098641

Ratcliff, W. C., Denison, R. F., Borrello, M., \& Travisano, M. (2012). Experimental evolution of multicellularity. Proceedings of the National Academy of Sciences of the United States of America, 109(5): 1595-1600.

https://doi.org/10.1073/pnas.1115323109

Reed, T. E., Robin, S. W., Schindler, D. E., Hard, J. J., \& Kinnison, M. T. (2010). Phenotypic plasticity and population viability: The importance of environmental predictability. Proceedings of the Royal Society B: Biological Sciences, 277(1699): 3391-3400. https://doi.org/10.1098/rspb.2010.0771

Rodríguez-Vargas, S., Sánchez-García, A., Martínez-Rivas, J. M., Prieto, J. A., \& RandezGil, F. (2007). Fluidization of membrane lipids enhances the tolerance of Saccharomyces cerevisiae to freezing and salt stress. Applied and Environmental Microbiology, 73(1): 110-116. https://doi.org/10.1128/AEM.01360-06

Rogers, T. R. (2002). Antifungal drug resistance: Does it matter? International Journal of Infectious Diseases, Suppl 1: S47-53. https://doi.org/10.1016/S1201-9712(02)90154-2 
Sanchez, Y., \& Lindquist, S. L. (1990). HSP104 required for induced thermotolerance.

Science, 248(4959): 1112-1115.

https://doi.org/10.1126/science.2188365

Sano, F., Asakawa, N., Inoue, Y., \& Sakurai, M. (1999). A dual role for intracellular trehalose in the resistance of yeast cells to water stress. Cryobiology, 39(1): 80-87. https://doi.org/10.1006/cryo.1999.2188

Sasse, C., Dunkel, N., Schäfer, T., Schneider, S., Dierolf, F., Ohlsen, K., \& Morschhäuser, J. (2012). The stepwise acquisition of fluconazole resistance mutations causes a gradual loss of fitness in Candida albicans. Molecular Microbiology, 86(3): 539-556. https://doi.org/10.1111/j.1365-2958.2012.08210.x

Schmitt, J., McCormac, A. C., \& Smith, H. (1995). A test of the adaptive plasticity hypothesis using transgenic and mutant plants disabled in phytochrome-mediated elongation responses to neighbors. American Naturalist, 146(6): 937-953. https://doi.org/10.1086/285832

Seger, J. (1987). What is bet-hedging? Oxford Surveys in Evolutionary Biology, 4: 182211. http://ci.nii.ac.jp/naid/10004816581/en/

Segrè, A. V., Murray, A. W., \& Leu, J. Y. (2006). High-resolution mutation mapping reveals parallel experimental evolution in yeast. PLoS Biology, 4(8): e256. https://doi.org/10.1371/journal.pbio.0040256 
Simons, A. M. (2002). The continuity of microevolution and macroevolution. Journal of Evolutionary Biology, 15(5): 688-701. https://doi.org/10.1046/j.14209101.2002.00437.x

Simons, A. M. (2009). Fluctuating natural selection accounts for the evolution of diversification bet hedging. Proceedings of the Royal Society B: Biological Sciences, 276(1664): 1987-1992. https://doi.org/10.1098/rspb.2008.1920

Simons, A. M. (2011). Modes of response to environmental change and the elusive empirical evidence for bet hedging. Proceedings of the Royal Society B: Biological Sciences, 278(1712), 1601-1609. https://doi.org/10.1098/rspb.2011.0176

Simons, A. M., \& Johnston, M. O. (2006). Environmental and genetic sources of diversification in the timing of seed germination: Implications for the evolution of bet hedging. Evolution, 60(11): 2280-2292. https://doi.org/10.1554/05-396.1

Spor, A., Wang, S., Dillmann, C., de Vienne, D., \& Sicard, D. (2008). “Ant” and "grasshopper" life-history strategies in Saccharomyces cerevisiae. PLoS ONE, 3(2): e1579. https://doi.org/10.1371/journal.pone.0001579

Stabell, O. B., Ogbebo, F., \& Primicerio, R. (2003). Inducible defences in Daphnia depend on latent alarm signals from conspecific prey activated in predators. Chemical Senses, 28(2): 141-153. https://doi.org/10.1093/chemse/28.2.141 
Stearns, S. C. (1989). Trade-Offs in Life-History Evolution. Functional Ecology, 3(3): 259268. https://doi.org/10.2307/2389364

Suzuki, G., Wang, Y., Kubo, K., Hirata, E., Ohnuki, S., \& Ohya, Y. (2018). Global study of holistic morphological effectors in the budding yeast Saccharomyces cerevisiae. BMC Genomics, 19(1): 149. https://doi.org/10.1186/s12864-018-4526-z

Tapia, H., Young, L., Fox, D., Bertozzi, C. R., \& Koshland, D. (2015). Increasing intracellular trehalose is sufficient to confer desiccation tolerance to Saccharomyces cerevisiae. Proceedings of the National Academy of Sciences of the United States of America, 112(19): 6122-6127. https://doi.org/10.1073/pnas.1506415112

Tarazona, E., García-Roger, E. M., \& Carmona, M. J. (2017). Experimental evolution of bet hedging in rotifer diapause traits as a response to environmental unpredictability. Oikos, 126(8): 1162-1172. https://doi.org/10.1111/oik.04186

Verghese, J., Abrams, J., Wang, Y., \& Morano, K. A. (2012). Biology of the heat shock response and protein chaperones: Budding yeast (Saccharomyces cerevisiae) as a model system. Microbiology and Molecular Biology Reviews, 76(2): 115-158. https://doi.org/10.1128/MMBR.05018-11

Verstraeten, N., Knapen, W. J., Kint, C. I., Liebens, V., Van den Bergh, B., Dewachter, L., Fu, Q., David, C. C., Fierro, A. C., Marchal, K., Beirlant, J., Versees, W., Hofkens, J., Jansen, M., Fauvart, M. \& Michiels, J. (2015). Obg and membrane depolarization 
are part of a microbial bet-hedging strategy that leads to antibiotic tolerance. Molecular Cell, 59(1): 9-21.

https://doi.org/10.1016/j.molcel.2015.05.011

Verweij, P. E., Snelders, E., Kema, G. H., Mellado, E., \& Melchers, W. J. (2009). Azole resistance in Aspergillus fumigatus: a side-effect of environmental fungicide use? The Lancet Infectious Diseases, 9(12): 789-795. https://doi.org/10.1016/S1473-3099(09)70265-8

Vilaprinyo, E., Alves, R., \& Sorribas, A. (2006). Use of physiological constraints to identify quantitative design principles for gene expression in yeast adaptation to heat shock. BMC Bioinformatics, 7:184.

https://doi.org/10.1186/1471-2105-7-184

Vincent, B. M., Lancaster, A. K., Scherz-Shouval, R., Whitesell, L., \& Lindquist, S. (2013). Fitness trade-offs restrict the evolution of resistance to Amphotericin B. PLoS Biology, 11(10): e1001692. https://doi.org/10.1371/journal.pbio.1001692

Vincenzi, S. (2014). Extinction risk and eco-evolutionary dynamics in a variable environment with increasing frequency of extreme events. Journal of the Royal Society Interface, 11(97): 20140441. https://doi.org/10.1098/rsif.2014.0441

Wallace-Salinas, V., \& Gorwa-Grauslund, M. F. (2013). Adaptive evolution of an industrial strain of Saccharomyces cerevisiae for combined tolerance to inhibitors and temperature. Biotechnology for Biofuels, 6: 151. 
https://doi.org/10.1186/1754-6834-6-151

Warringer, J., \& Blomberg, A. (2003). Automated screening in environmental arrays allows analysis of quantitative phenotypic profiles in Saccharomyces cerevisiae. Yeast, 20(1): 53-67. https://doi.org/10.1002/yea.931

Weis, A. E., \& Gorman, W. L. (1990). Measuring selection on reaction norms:An exploration of the Eurosta-solidago System. Evolution, 44(4): 820-831. https://doi.org/10.2307/2409548

White, E. B., DeBach, P., \& Garber, M. J. (1970). Artificial selection for genetic adaptation to temperature extremes in Aphytis lingnanensis Compere (Hymenoptera: Aphelinidae) . Hilgardia, 40(6): 161-192. https://doi.org/10.3733/hilg.v40n06p161

Yoshikawa, K., Tanaka, T., Ida, Y., Furusawa, C., Hirasawa, T., \& Shimizu, H. (2011). Comprehensive phenotypic analysis of single-gene deletion and overexpression strains of Saccharomyces cerevisiae. Yeast, 28(5): 349-361. https://doi.org/10.1002/yea.1843

Yoshimura, J., Tanaka, Y., Togashi, T., Iwata, S., \& Tainaka, K. ichi. (2009). Mathematical equivalence of geometric mean fitness with probabilistic optimization under environmental uncertainty. Ecological Modelling, 220(20): 2611-2617. https://doi.org/10.1016/j.ecolmodel.2009.06.046 
Zakrzewska, A., van Eikenhorst, G., Burggraaff, J. E. C., Vis, D. J., Hoefsloot, H., Delneri, D., Oliver, S. G., Brul, S. \& Smits, G. J. (2011). Genome-wide analysis of yeast stress survival and tolerance acquisition to analyze the central trade-off between growth rate and cellular robustness. Molecular Biology of the Cell, 22(22): 4435-4446. https://doi.org/10.1091/mbc.E10-08-0721

Zammuto, R. M., \& Millar, J. S. (1985). A Consideration of Bet-Hedging in Spermophilus columbianus. Journal of Mammalogy, 66(4): 652-660. https://doi.org/10.2307/1380791

Zeevi, D., Lubliner, S., Lotan-Pompan, M., Hodis, E., Vesterman, R., Weinberger, A., \& Segal, E. (2014). Molecular dissection of the genetic mechanisms that underlie expression conservation in orthologous yeast ribosomal promoters. Genome Research, 24(12): 1991-1999. https://doi.org/10.1101/gr.179259.114

Zeldovich, K. B., Chen, P., \& Shakhnovich, E. I. (2007). Protein stability imposes limits on organism complexity and speed of molecular evolution. Proceedings of the National Academy of Sciences of the United States of America, 104(41): 16152-16157. https://doi.org/10.1073/pnas.0705366104

Zhang, Q., Sahin, O., McDermott, P. F., \& Payot, S. (2006). Fitness of antimicrobialresistant Campylobacter and Salmonella. Microbes and Infection, 8(7): 1972-1978. https://doi.org/10.1016/j.micinf.2005.12.031 